\title{
Glycogen Synthase Kinase 3 Regulates the Genesis of Displaced Retinal Ganglion Cells3
}

\author{
Elena Kisseleff, ${ }^{1}$ Robin J. Vigouroux, ${ }^{3}$ Catherine Hottin, ${ }^{1}$ Sophie Lourdel, ${ }^{1}$ Leah Thomas, ${ }^{1}$ Parth Shah, ${ }^{2}$ \\ Alain Chédotal, ${ }^{3}$ - Muriel Perron, ${ }^{1}$ (D)Anand Swaroop, ${ }^{2}$ and ${ }^{\circledR}$ Jerome E. Roger ${ }^{1,2}$
}

\author{
https://doi.org/10.1523/ENEURO.0171-21.2021
}

\begin{abstract}
${ }^{1}$ Paris-Saclay Institute of Neuroscience, CERTO-Retina France, CNRS, Université Paris-Saclay, 91400, Saclay, France, ${ }^{2}$ Neurobiology-Neurodegeneration and Repair Laboratory, National Eye Institute, National Institutes of Health, Bethesda, Maryland 20892, and ${ }^{3}$ UPMC Université Paris 06, INSERM, CNRS, Institut de la Vision, Sorbonne Universités, 75012 Paris, France
\end{abstract}

\begin{abstract}
Glycogen synthase kinase 3 (GSK3) proteins (GSK3 $\alpha$ and GSK3 $\beta$ ) are key mediators of signaling pathways, with crucial roles in coordinating fundamental biological processes during neural development. Here we show that the complete loss of GSK3 signaling in mouse retinal progenitors leads to microphthalmia with broad morphologic defects. A single wild-type allele of either Gsk3 $\alpha$ or Gsk3 $\beta$ is able to rescue this phenotype. In this genetic context, all cell types are present in a functional retina. However, we unexpectedly detected a large number of cells in the inner nuclear layer expressing retinal ganglion cell (RGC)-specific markers (called displaced RGCs, dRGCs) when at least one allele of Gsk3 $\alpha$ is expressed. The excess of dRGCs leads to an increased number of axons projecting into the ipsilateral medial terminal nucleus, an area of the brain belonging to the non-image-forming visual circuit and poorly targeted by RGCs in wild-type retina. Transcriptome analysis and optomotor response assay suggest that at least a subset of dRGCs in Gsk3 mutant mice are direction-selective RGCs. Our study thus uncovers a unique role of GSK3 in controlling the production of ganglion cells in the inner nuclear layer, which correspond to dRGCs, a rare and poorly characterized retinal cell type.
\end{abstract}

Key words: cell death; displaced ganglion cells; glycogen synthase kinase 3; post-translational modifications; medial terminal nucleus; retinal development

\section{Significance Statement}

Glycogen synthase kinase 3 (GSK3) proteins (GSK3 $\alpha$ or GSK3 $\beta$ ) are key mediators of signaling pathways, especially in the CNS but poorly described in the retina. Here we show that the complete loss of GSK3 in mouse retinal progenitors leads to microphthalmia. However, when only one allele of Gsk3 $\alpha$ or Gsk3 $\beta$ is present, all cell types are present with a functional retina. Interrestingly, we unexpectedly uncovered a unique role of GSK3s in controlling the genesis of retinal ganglion cells in the inner nuclear layer, which could correspond to a rare and poorly characterized retinal cell type. Therefore, our mouse models potentially offer a unique and powerful model system to study the visual function of displaced retinal ganglion cells in mammals.

Received April 19, 2021; accepted September 2, 2021; First published September 13, 2021.

The authors declare no competing financial interests.

Author contributions: E.K., R.J.V., C.H., A.C., M.P., A.S., and J.E.R. designed research; E.K., R.J.V., C.H., S.L., L.T., P.S., and J.E.R. performed research; E.K., R.J.V., C.H., S.L., L.T., M.P., A.S., and J.E.R. analyzed data; E.K., R.J.V., M.P., A.S., and J.E.R. wrote the paper.

\section{Introduction}

Glycogen synthase kinase $3 \alpha$ (GSK3 $\alpha$ ) and $\beta$ (GSK3 $\beta$ ) are functionally redundant serine/threonine kinases encoded by two different genes, sharing $95 \%$ identity in their kinase domain (Doble et al., 2007). GSK3s exist at the crossroads of multiple signaling pathways and act as a key molecular switch to mediate their output and guide distinct cellular processes (Doble and Woodgett, 2003; 
Espinosa et al., 2003; Wang and Li, 2006; Shimizu et al., 2008; Jin et al., 2009; Cole, 2012). Among the signaling pathways regulated by GSK3s, the Wnt canonical pathway is the most well described, with GSK3 $\beta$ inhibition triggering an increase in $\beta$-catenin protein levels and its nuclear translocation to activate target gene expression (Doble and Woodgett, 2003).

GSK3 is a key regulator of neural stem/precursor cell proliferation in developing as well as adult brain (Eom and Jope, 2009; Kim et al., 2009; Hur and Zhou, 2010; Pachenari et al., 2017). Conditional deletion and gain-offunction experiments indicate that GSK3 promotes neuronal differentiation (Kim et al., 2009; Hur and Zhou, 2010). GSK3 exerts its effects through the phosphorylation of key proteins involved in neural development, including proneural factors such as Neurogenin 2 and NeuroD (Moore et al., 2002; Li et al., 2012). In addition, GSK3 finetunes the balance between cell death and survival, and its altered function is associated with neurodegenerative pathologies including Alzheimer's disease, bipolar disorders, and Parkinson's disease (Kremer, 2011; Medina et al., 2011; Jacobs et al., 2012; Li et al., 2014; Maurer et al., 2014; Golpich et al., 2015).

GSK3 proteins are widely expressed in the developing retina (Pérezleón et al., 2013). GSK3-dependent phosphorylation was shown to control the timing of proneural factor activity and thereby regulate retinal cell fate determination. For instance, the inhibition of GSK3 signaling in the developing Xenopus retina leads to increase in earlyborn cell types at the expense of late-born cells (Marcus et al., 1998; Moore et al., 2002).

To elucidate GSK3 function in mammalian retina development, we generated conditional loss-of-function alleles of Gsk $3 \alpha$ and Gsk3 $\beta$ in retinal progenitor cells (RPCs). We showed that complete loss of both GSK3s severely impacts retinal morphology with microphthalmia phenotype, which could be completely rescued with the expression of just one Gsk3 $\alpha$ or Gsk3 $\beta$ wild-type (WT) allele. We also noted the presence of a large number of displaced retinal ganglion cells (dRGCs) in the inner nuclear layer (INL) in the absence of either Gsk3 $\alpha$ or Gsk3 $\beta$. In normal conditions, this is a rare retinal cell subtype, poorly characterized so far. Anterograde labeling of the axonal ganglion cell projections into the brain of Gsk3 mutant mice, allowed us to further support their dRGCs identity. Our study thus identifies GSK3 as a

This research was supported by the CNRS, Retina France, DIM gene therapy, Fondation Valentin Haüy, and by the Intramural Research Program of the National Eye Institute (Grants EY-000450 and EY-000546). EK was supported by the Ernst Ludwig Ehrlich Studienwerk.

Acknowledgements: We thank Dr. Jim Woodgett for providing the Gsk3 floxed lines, and Tudor Badea and Peter Gruss for the $\alpha$-Cre line. We also thank Elodie-Kim Grellier, Yide Mi, and Jessica Gumerson for their help with mouse colonies and technical support.

Correspondence should be addressed to Jerome E. Roger at jerome. roger@universite-paris-saclay.fr; Anand Swaroop at swaroopa@nei.nih.gov; or Muriel Perron at muriel.perron@universite-paris-saclay.fr.

https://doi.org/10.1523/ENEURO.0171-21.2021

Copyright ( $\odot 2021$ Kisseleff et al.

This is an open-access article distributed under the terms of the Creative Commons Attribution 4.0 International license, which permits unrestricted use, distribution and reproduction in any medium provided that the original work is properly attributed. possible determinant of dRGC genesis. We also provide transcriptomic data and visual tests, suggesting that at least a subset of these supernumerary dRGCs in Gsk3 mutant retinas are direction-selective RGCs.

\section{Materials and Methods}

\section{Animals and tissue collection}

All animal experiments have been conducted in accordance with the European Communities Council Directive of 22 September 2010 (2010/63/EEC), the effective European Union guidelines, and the Association for Research in Vision and Ophthalmology statement for the use of animals in ophthalmic and visual research. All animal care and experimentation were also conducted in accordance with guidelines, under license APAFIS\#1018-2016072611404304 granted by Institutional Animal Care Committee 059 in France and by the Animal Care and Use Committee at the National Institutes of Health (license ASP\#650). Gsk3 $\alpha$ and Gsk3 $\beta$ floxed mice were generously provided by Jim Woodgett (University of Toronto, Toronoto, ON, Canada). Floxed Gsk3 mice were mated with those carrying the retina-specific regulatory element of murine Pax6 driving the expression of the Cre recombinase $(\alpha-\mathrm{Cre})$ in retinal progenitors as early as embryonic day 10.5 (E10.5) generously provided by Peter Gruss, Max-PlanckInstitute of Biophysical Chemistry, Göttingen, Germany (Marquardt et al., 2001). Mice are on a mixed background C57BL/6J and 129/SvJ. Animals from either sex were used for experimental procedures. All mouse genotyping was performed as described previously (Hamon et al., 2019).

\section{Hematoxylin and eosin staining and immunostaining}

Methacrylate sections were used for hematoxylin and eosin (H\&E) staining as previously described (Hamon et al., 2019). For immunohistochemistry $(\mathrm{IHC})$ on frozen sections, enucleated eyeballs were fixed at the required stage in 4\% PFA for $60 \mathrm{~min}$ on ice and incubated in increasing concentrations of sucrose $(10 \%, 20 \%$, and $30 \%)$, then embedded in Optimal Cutting Temperature compound. Embedded eyeballs were serially cut into $12 \mu \mathrm{m}$ sections using a cryostat. For embryonic stages, pregnant females were sacrificed and whole heads of pups were harvested in paraffin. $\mathrm{IHC}$ was performed as described previously (Roger et al., 2012). Primary and secondary antibodies are listed in Table 1. Sections were counterstained with 1:1000 DAPI $\left(4^{\prime}, 6^{\prime}\right.$-diamidino-2-phenylindole dihydrochloride; $1 \mathrm{mg} / \mathrm{ml}$; Thermo Fisher Scientific).

\section{5-Ethynyl-2' -deoxyuridine labeling and terminal deoxynucleotidyl transferase-mediated biotinylated UTP nick end labeling assay}

For 5-ethynyl-2' -deoxyuridine (EdU) labeling, females were injected intraperitoneally with $10 \mathrm{~mm}$ of EdU (Thermo Fisher Scientific). EdU incorporation was detected on paraffin sections or frozen sections using the Click-iT EdU Imaging Kit following manufacturer recommendations (Thermo Fisher Scientific). Apoptosis was detected by TUNEL (terminal deoxynucleotidyl transferase-mediated biotinylated UTP nick end labeling) assays using in situ cell death detection kit 
Table 1: List of primary and secondary antibodies used for immunohistochemistry and western blot

\begin{tabular}{lllll}
\hline & & & & \\
Antigen & Host & Supplier & Catalog no. & Dilution \\
Primary antibodies & & & & WB \\
$\alpha$-Tubulin & Mouse & Sigma-Aldrich & T5168 & $1: 200.000$ \\
GSK3 $/ \beta$ & Mouse & Thermo Fisher Scientific & $44-610$ & $1: 1000$ \\
GSK3 $\beta$ & Mouse & BD & 610201 & $1: 250$ \\
Brn3a & Mouse & Santa Cruz Biotechnology & sc-8429 & $1: 250$ \\
Calbindin D-28k & Rabbit & Swant & 300 & $1: 100$ \\
Calretinin & Mouse & EMD Millipore & MAB1568 & $1: 1000$ \\
Cone arrestin & Rabbit & EMD Millipore & AB15282 & $1: 1000$ \\
Rhodopsin & Mouse & Abcam & MAB5316 & $1: 2000$ \\
Tbr2 & Rat & Thermo Fisher Scientific & $14-4876$ & $1: 300$ \\
Foxp2 & Goat & Santa Cruz Biotechnology & sc-21069 & $1: 1000$ \\
Rbpms & Rabbit & PhosphoSolutions & $1830-R B P M S$ & $1: 400$ \\
CHAT & Goat & Millipore & AB144P & $1: 100$ \\
Secondary antibodies & & & \\
Alexa Fluor-555 anti-mouse IgG2A & Goat & Thermo Fisher Scientific & A21127 & $1: 1000$ \\
Alexa Fluor-555 anti-mouse IgG2B & Goat & Thermo Fisher Scientific & A21147 & $1: 1000$ \\
Alexa Fluor-488 anti-rabbit & Donkey & Thermo Fisher Scientific & A21206 & $1: 1000$ \\
Alexa Fluor-488 anti-mouse IgG1 & Goat & Thermo Fisher Scientific & A21240 & $1: 1000$ \\
Alexa Fluor-488 anti-rabbit & Goat & Thermo Fisher Scientific & A21244 & $1: 1000$ \\
HRP anti-mouse IgG & Goat & Sigma-Aldrich & A4416 \\
Alexa Fluor-488 anti-goat & Donkey & Thermo Fisher Scientific & A11055 \\
\hline
\end{tabular}

WB, Western blot; IHC, Immunohistochenistry.

(Promega). All images were acquired using a confocal microscope (model LSM710, Zeiss) and Zen software (Zeiss).

\section{Immunoblotting}

Frozen retinas were lysed by sonication in lysis buffer (20 $\mathrm{mm} \mathrm{Na}_{2} \mathrm{HPO}_{4}, 250 \mathrm{~mm} \mathrm{NaCl}, 30 \mathrm{~mm} \mathrm{NaPPi}, 0.1 \%$ NP40, 5 mм EDTA, 5 mm DTT) supplemented with protease inhibitor cocktail (Sigma-Aldrich). Lysates concentration was determined using a Lowry protein assay kit (BIO-RAD) following sonication and centrifugation. Protein supernatants were separated under denaturing conditions by SDS-PAGE, transferred onto nitrocellulose membrane, and probed with antibodies (Table 1), as described (Roger et al., 2010). Proteins were visualized using enhanced chemiluminescence kit (BIO-RAD). $\alpha$-Tubulin was used for normalization. Quantification was performed using ImageJ software (http://imagej.nih.gov/ $\mathrm{ij} /$; provided in the public domain by $\mathrm{NIH}$ ).

\section{Retinal flat mount}

Fixed retinas were permeabilized and blocked in a solution containing $0.5 \%$ Triton $\mathrm{X}-100,5 \%$ normal donkey serum, $1 \times$ PBS, $0.1 \mathrm{~g} / \mathrm{L}$ thimerosal for $1 \mathrm{~d}$ at room temperature $(\mathrm{RT})$ under agitation. Primary antibodies were diluted in a solution containing $0.5 \%$ Triton $X-100,5 \%$ normal donkey serum, 10\% dimethylsulfoxide, $1 \times$ PBS, and $0.1 \mathrm{~g} / \mathrm{L}$ thimerosal for 3 days at RT under agitation. The retinas were then washed for $1 \mathrm{~d}$ in PBST $(1 \times$ PBS, $0.5 \%$ Triton $X-100)$. Secondary antibodies were diluted in the same solution as primary antibodies and left for $2 \mathrm{~d}$. After retinas were washed for $1 \mathrm{~d}$, they were flat-mounted on slides and imaged using a scanning confocal microscope (model FV1000, Olympus). Primary and secondary antibodies are listed in Table 1.

\section{Electroretinography}

Electroretinogram (ERG) recordings were performed using a focal ERG module attached to a retinal imaging microscope (model Micron IV, Phoenix Technology Group). Briefly, mice were dark adapted overnight and prepared for the experiment under dim red light. The mice were anesthetized with ketamine $(100 \mathrm{mg} / \mathrm{kg})$ and xylazine $(10 \mathrm{mg} / \mathrm{kg})$ and received topical proparacaine hydrochloride (0.5\%; Alcon) via eye drops. Pupils were dilated with tropicamide (1\%; Alcon) and phenylephrine (2.5\%; Alcon) and were lightly coated with GONAK hypromellose ophthalmic demulcent solution (2.5\%; Akorn). The lens of the Micron IV was placed directly on the cornea, and a reference electrode was placed on the mouse head. Scotopic responses were elicited with a series of flashes of increasing light intensities from -1.7 to $2.2 \mathrm{~cd} / \mathrm{s} / \mathrm{m}^{2}$. Photopic responses were elicited under roddesensitizing background light with a series of flashes of increasing light intensities from -0.5 to $2.8 \mathrm{~cd} / \mathrm{s} / \mathrm{m}^{2}$. Values of a- and b-wave were extracted and plotted for comparisons between groups of interest.

\section{Optomotor response}

Real-time video tracking and automated measurements of compensatory head movements in freely moving mice were performed using an optomotor response (OMR) recording setup (PhenoSys; Kretschmer et al., $2013,2015)$. Each mouse was placed on a platform in the center of four computer-controlled LCD monitors. Visual stimuli were sinusoidally modulated luminance gratings generated by four LCD screens $(60 \mathrm{~Hz}$ refresh rate; OkrArena, PhenoSys), presented with a constant rotation. Video tracking considered the distance of the animal from the monitors, thereby keeping the spatial frequency of the retinal image constant and providing data for automated OMR quantifications. 
OMRs were recorded using two different Michelson contrasts and different spatial frequencies (presented in random order) in the following two mouse groups: $100 \%$ contrast or $50 \%$ contrast $\left(n=13\right.$ for Gsk $3 \alpha^{f /+} \beta^{f / f}$ and 18 for $\mathrm{Gsk} 3 \alpha^{f /+} \beta^{f / f} ; \alpha$-Cre genotype). All stimuli were presented for $60 \mathrm{~s}$ randomly in either clockwise or counterclockwise direction. The measurements were completed in three trials for each animal. At $100 \%$ and $50 \%$ contrast, OMRs were recorded in response to sinusoidal gratings at 12 spatial frequencies between 0.0125 and 0.5 cycles per degree (cpd). The number of head movements recorded at a speed range from 2 to $14 \%$ in the same direction as the stimulus ( $T_{\text {_CCorrect) }}$ and in the opposite direction ( $T$ Incorrect) were used to calculate the OMR indices ( $T_{-}$Correct $/ T_{-}$Incorrect) at each spatial frequency.

\section{Retrograde labeling of retinal ganglion cells}

For retrograde labeling, eyes were enucleated with a piece of the optic nerve and fixed in 4\% PFA for $30 \mathrm{~min}$. Rhodamine B isothiocyanate-dextran (Sigma-Aldrich) was applied on the top of the optic nerve and incubated for $60 \mathrm{~min}$. Eyes were flat-mounted after the remaining dye was washed out for $48 \mathrm{~h}$ in PBS at $4^{\circ} \mathrm{C}$. The $z$ series images were acquired using a confocal microscope (model SP5, Leica Biosystems), and 3D reconstruction was performed using Volocity (PerkinElmer).

\section{Anterograde labeling of retinal ganglion cell projections}

Anterograde labeling

For anterograde tracing of retinal projections, a cholera toxin $\beta$ subunit (CTB) was used. Animals were anesthetized using a cocktail of ketamine $(60 \mathrm{mg} / \mathrm{kg})$ and xylazine $(10 \mathrm{mg} / \mathrm{kg})$, and a subsequent bilateral injection of $1.2 \mu \mathrm{l}$ of CTB at $1 \mathrm{mg} / \mathrm{ml}$ coupled with either Alexa Fluor-555 or Alexa Fluor-647 (Beckman Coulter Life Sciences) was performed intravitreally. Three days following the injection, mice were perfused with $4 \%$ PFA.

\section{Tissue clearing and 3D imaging}

For 3D imaging of CTB-labeled brains, a methanol clearing protocol was conducted using modification from the iDISCO+ protocol (Belle et al., 2014, 2017). Briefly, brains were dehydrated by immersion in progressive baths of methanol $/ 1 \times$ PBS $(20 \%, 40 \%, 60 \%, 80 \%, 100 \%, 100 \%)$ for $2 \mathrm{~h}$ each at RT on a tube rotator (model SB3, Stuart) at $14 \mathrm{rpm}$, using a $15 \mathrm{ml}$ centrifuge tube (model TPP, Dutcher) protected from light. Following these baths, samples were immersed overnight in two-thirds cubic meter dichloromethane (DCM; Sigma-Aldrich) and then were given a $30 \mathrm{~min}$ bath in $100 \%$ DCM before being transferred in dibenzyl ether (Sigma-Aldrich) overnight prior imaging.

3D imaging/image acquisition for all samples was performed as described previously (Belle et al., 2014, 2017). Acquisitions were performed using a microscope (UltraMicroscope I, LaVision BioTec) with ImspectorPro software (LaVision Biotec). The step size between each image was fixed at $2 \mu \mathrm{m}$ with a numerical aperture of 0.120 and $150 \mathrm{~ms}$ acquisition using a PCO Edge
SCMOS CCD camera $(2560 \times 2160$ pixel size; LaVision BioTec).

\section{Image analysis}

Imaris x64 software (version 9.1.2; Bitplane) was used for all image analysis. Stack images were first converted from .tiff to .ims files using the Imaris file converter version 9.1.2. 3D reconstruction was visualized with the "volume rendering" function. To isolate ipsilateral and contralateral medial terminal nucleus (MTN) volumes, manual segmentation was conducted using the "surface" tool and the isoline selection (density, 10\%). Each ipsilateral and contralateral projection of the MTN was segmented to generate a volume (cubic micrometer). Movie reconstruction with .tiff series was performed with ImageJ (1.50e, Java 1.8.0_60, 64-bit) and iMovie (version 10.1.1).

\section{Whole-transcriptome sequencing and analysis}

Whole-transcriptome sequencing was performed on three independent biological replicates from Gsk3 $\alpha^{f /+} \beta^{f / f}$; $\alpha$-Cre and Gsk3 $\alpha^{f /+} \beta^{f / f}$ retinas at postnatal day 60 (P60). After harvesting, both retinas for each animal were immediately frozen. RNA was extracted using a Nucleospin RNA Plus Kit (Macherey-Nagel). RNA quality and quantity were evaluated using a BioAnalyzer 2100 with RNA 6000 Nano Kit (Agilent Technologies). Stranded RNA sequencing (RNASeq) libraries were constructed from $100 \mathrm{ng}$ high-quality total RNA (RNA integrity number, $>8$ ) using the TruSeq Stranded mRNA Library Preparation Kit (Illumina). Pairedend sequencing of 40 base lengths was performed on a NextSeq 500 system (Illumina). Pass-filtered reads were mapped using STAR and aligned to mouse reference genome GRCm38.94 (Dobin et al., 2013). A count table of the gene features was obtained using FeatureCounts (Liao et al., 2014). Normalization, differential expression analysis and FPKM (fragments per kilobase of exon per million fragments mapped) values were computed using EdgeR (Chen et al., 2014). An FPKM filtering cutoff of 1 in at least one of the six samples was applied. A false discovery rate (FDR) of $\leq 0.05$ was considered significant and a fold change (FC) cutoff of 1.5 was applied to identify differentially expressed genes (DEGs). Comprehensive gene list analysis, enriched biological pathways, and gene annotation were based on the Gene Ontology classification system using Metascape (Zhou et al., 2019). Data visualization was done using the GOplot R package (Walter et al., 2015). To evaluate the expression of the DEGs in RGCs, we used published whole-transcriptome analysis from purified RGCs that are available on the Gene Expression Omnibus database (GSE87647; Sajgo et al., 2017).

\section{Gene expression analysis by real-time PCR}

After RNA extraction using the Nucleospin RNA Plus Kit (Macherey-Nagel), $500 \mathrm{ng}$ of total RNA was reverse transcribed using the iScript cDNA Synthesis Kit according to manufacturer instructions (BIO-RAD). Primers used for quantitative real-time PCR (qRT-PCR) are shown in Table 2. For each qRT-PCR, $2 \mu \mathrm{l}$ of a 10-fold dilution of synthetized cDNA was used, and the reactions were performed in 
Table 2: List of primers used for qRT-PCR

\begin{tabular}{|c|c|c|}
\hline Gene name & Primer forward & Primer reverse \\
\hline$\overline{\text { Cartpt }}$ & 5'-TAAAGTTTGCGTTCCCCTCAG-3' & 5'-CAACACCATTCGAGGCATTCT-3' \\
\hline Epha2 & 5'-GACCTCCCCATCTTCATTTGG-3' & 5'-GCGTACAGTGCCCTAGTCATA-3' \\
\hline Chrna5 & 5'-CTTGAGTACCAACACTGTCCG-3' & 5'-CCAGTACTCCAAAGATGCCCT-3' \\
\hline Chrna2 & 5'-CATTATCGTCTGCTTCCTGGG-3' & 5'-CTTGGAGCCAACATGAGGGA-3' \\
\hline Chrna7 & 5'-CTGTAGCTGTCGGTCTTGAGA-3' & 5'-CAATGATATGCCGGTGATGGG-3' \\
\hline
\end{tabular}

technical triplicates on a C1000 thermal cycler (model CFX96 Real-Time System, BIO-RAD) using SsoFast EvaGreen Supermix (BIO-RAD) as previously described (Hamon et al., 2019). qRT-PCR experiments were performed on three independent biological replicates. Differential expression was determined using the $\Delta \Delta \mathrm{Ct}$ method with the geometric average of Rps26 and Srp72 as an endogenous control (Livak and Schmittgen, 2001).

\section{Statistical analysis}

Statistical analyses were performed with GraphPad Prism version 8.3.0 (GraphPad Software). Results are reported as the mean \pm SEM. A nonparametric Mann-Whitney $U$ test was used to analyze cell counting and qPCR data. A $p$ value of $\leq 0.05$ was considered significant. For OMR assay statistical analysis, a Grubbs' test was performed at 5\% to remove outliers followed by two-way ANOVA (genotype and spatial frequency) with Bonferroni post hoc test. A $p$ value $\leq 0.05$ was considered significant.

\section{Results}

Retinal progenitor-specific deletion of both Gsk3 $\alpha$ and Gsk3 $\beta$ results in microphthalmia

We crossed the floxed Gsk $3 \alpha^{f / f} \beta^{f / f}$ mice with $\alpha$-Cre ( $\alpha$ Pax6-Cre) line to generate Gsk3 $\alpha^{f / f} \beta^{f / f} ; \alpha$-Cre mice in which Gsk3 deletion occurs only in retinal progenitors as early as E10.5 (Marquardt et al., 2001). We first validated our model by assessing the efficacy of Gsk3 $\alpha$ and Gsk3 $\beta$ deletion at E12.5. IHC using an antibody recognizing both GSK3 proteins showed ubiquitous expression in control retinas (Fig. $1 A)$. Both Gsk3 genes were efficiently deleted in the peripheral retina of Gsk3 $\alpha^{f / f} \beta^{f / f} ; \alpha$-Cre mice, but their expression in the central retina remained preserved consistent with the previously described $\alpha$-Cre expression pattern (Marquardt et al., 2001).

H\&E staining revealed major morphologic defects with profound retinal disorganization, including the loss of radial arrangement as well as folds and aggregates of $\mathrm{RPCs}$, in Gsk3 $\alpha^{f / f} \beta^{f / f} ; \alpha$-Cre retina as early as E12.5 (Fig. $1 B)$. In addition, blood was detected inside the retinal neuroblastic layer. The structure of the retina worsened rapidly during development, although the central part remained unperturbed, consistent with continued Gsk3 expression in this region. At and after E14.5, the retina was largely reduced, whereas the eye size itself was comparable to those of littermate controls (Fig. $1 B)$. A large quantity of blood accumulated inside the eyeball at P2. Finally, growth of the eyeball was severely reduced, leading to microphthalmia in the adult (data not shown).

\section{Multiple allelic combinations revealed functional

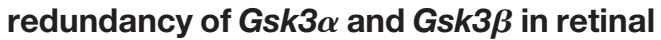 development}

Severe deleterious effects by the loss of both Gsk3 $\alpha$ and $\mathrm{Gsk} 3 \beta$ in RPCs in early development preclude the analysis of late retinal histogenesis. To circumvent this, we generated animals with different combinations of Gsk3 deletion (loss of only one Gsk3 gene: Gsk3 $\alpha^{f / f}$ $\beta^{+/+} ; \alpha$-Cre or Gsk3 $\alpha^{+/+} \beta^{\text {f/f }} ; \alpha$-Cre, or three-quarter deletion: Gsk3 $\alpha^{f / f} \beta^{f /+} ; \alpha$-Cre or Gsk3 $\alpha^{f /+} \beta^{f / f} ; \alpha$-Cre). Immunoblot analysis using anti-GSK3 antibody (recognizing both proteins) in 2-month-old animals with different combinations of Gsk3 $\alpha$ and Gsk3 $\beta$ floxed alleles demonstrated the efficacy of Gsk3 $\alpha$ and Gsk3 $\beta$ deletion in areas where the Cre recombinase was expressed during early retinal development (all retinal progenitors with the exception of a stripe located in the dorsocentral region (Marquardt et al., 2001; Fig. 2A). IHC analysis using anti-GSK3 $\beta$ showed ubiquitous expression of Gsk3 $\beta$ in adult control retina and its complete loss in Gsk3 $\alpha^{f /+} \beta^{f / f} ; \alpha$-Cre retina (Fig. 2B). At 2 months, retinal histology revealed the correct laminated architecture with normal photoreceptors and interneurons when even one allele of Gsk3 $\alpha$ (Fig. 2C,D) or Gsk3 $\beta$ (data not shown) was present. Photopic and scotopic ERG recordings, corresponding to cone and rod function, respectively, did not show any significant difference between Gsk3 $\alpha^{f /+} \beta^{f / f} ; \alpha$-Cre and control retina (Fig. 2E,F). These results were similar in mice carrying any combination of Gsk3 deletion (data not shown). We therefore conclude that a single allele of wild-type Gsk3 $\alpha$ or Gsk3 $\beta$ is sufficient to rescue obvious structural and functional defects in the complete absence of GSK3 signaling.

\section{Loss of either Gsk3 $\alpha$ or Gsk3 $\beta$ in RPCs leads to increased number of displaced retinal ganglion cells}

Although a single allele of either Gsk3 $\alpha$ or Gsk3 $\beta$ permitted normal retinal development (Fig. 2), we observed a striking increase in the number of RGCs, as indicated by Brn3a-positive cells, in the INL of Gsk3 $\alpha^{f /+} \beta^{f / f} ; \alpha$-Cre retina compared with controls (Fig. $3 A$ ). Brn3a-positive cells in the INL have been described as dRGCs, a rare cell type in the mammalian retina (Buhl et al., 1988; Doi et al., 1994). All Brn3a-positive cells in the INL of Gsk3 $\alpha^{f /+}$ $\beta^{f / f} ; \alpha$-Cre retina also expressed NF68 that labels cell bodies 
A
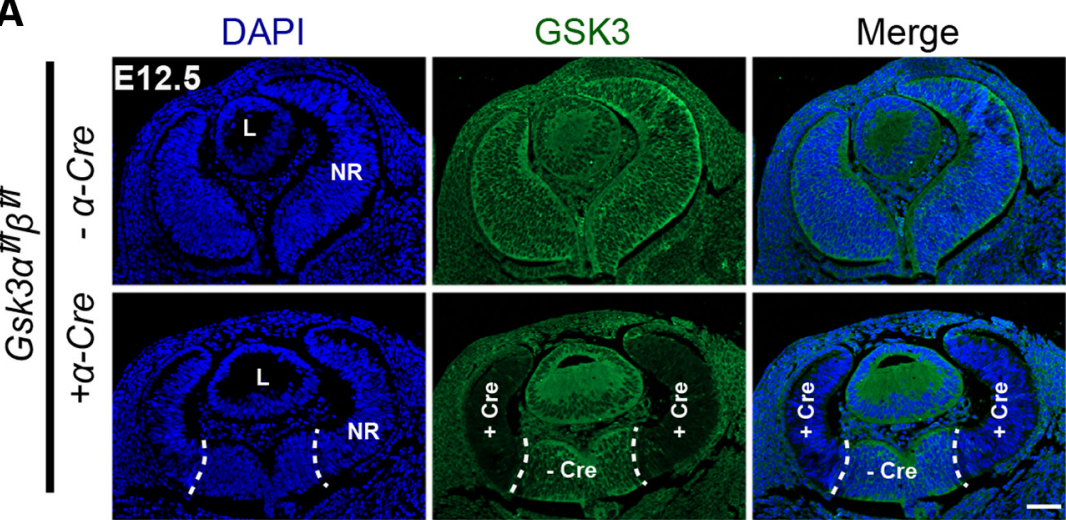

B

E12.5

E14.5

P2

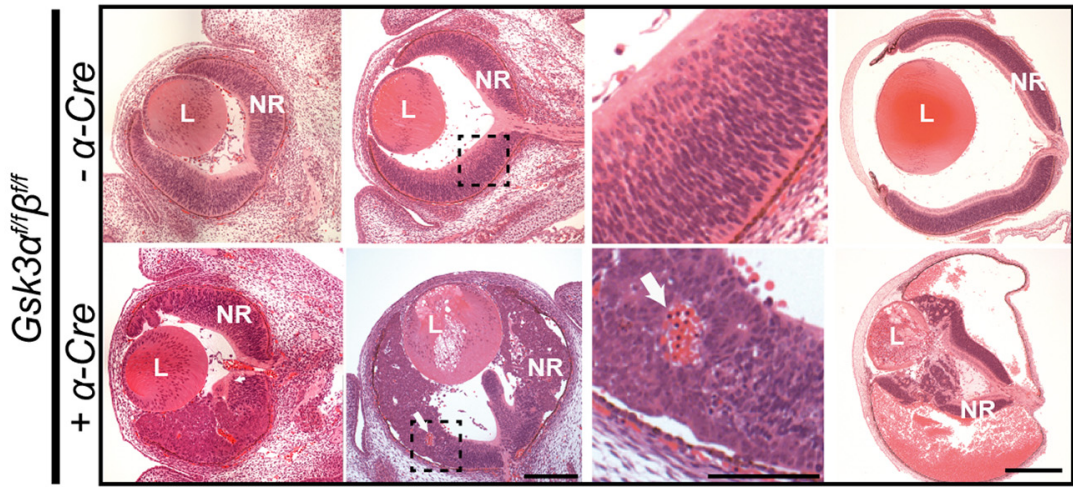

Figure 1. Developmental defects and microphthalmia in Gsk3-deficient retina with aberrant nuclear translocation of $\beta$-catenin, a key effector of the Wnt canonical pathway. A, IHC of E12.5 retinas from Gsk3 $\alpha^{f / f} \beta^{f / f}$ mice expressing or not $\alpha$-Cre using a pan-GSK3 antibody (green) shows efficient deletion at the periphery where the Cre expression has been previously reported (delimited by dashed line). Scale bar, $100 \mu \mathrm{m}$. B, H\&E staining on methacrylate sections at E12.5, E14.5, and P2 reveals large retinal morphogenesis defects in Gsk3 $\alpha^{f / f} \beta^{f / f} ; \alpha$-Cre with blood invasion into the eyeball (showed by white arrow). L, Lens; NR, neural retina. Scale bars: E12.5 and E14.5, $100 \mu \mathrm{m}$; P2, $500 \mu \mathrm{m}$; E14.5, right, magnified image, $50 \mu \mathrm{m}$.

and axons of RGCs (Fig. 3A). To validate that these Brn3apositive cells in the INL were indeed RGCs with axonal projections included in the optic nerve, we performed retrograde labeling with rhodamine-dextran applied onto the optic nerve of Gsk3 $\alpha^{f /+} \beta^{f / f} ; \alpha$-Cre mice. Subsequent 3D reconstructions on flat-mounted retinas revealed the presence of numerous fluorescent cell bodies located in the INL compared with controls (Fig. 3B) demonstrating that axons of dRGCs indeed reached the optic nerve. Thus, Brn3a-positive cells located in the INL of Gsk3 $\alpha^{f /+} \beta^{f / f} ; \alpha$-Cre retinas are indeed RGCs.

Increased dRGCs were observed in retinas carrying any combination of Gsk3 deletions tested (Gsk3 $\alpha^{f / f} \beta^{+/+}$, Gsk3 $\alpha^{+/+} \beta^{f / f}$, Gsk3 $\alpha^{f /+} \beta^{f / f}$, or Gsk3 $\alpha^{f / f} \beta^{f /+}$ ), with the highest number detected in Gsk3 $\alpha^{f /+} \beta^{f / f} ; \alpha$-Cre mice compared with controls (10-fold increase; Fig. $3 C$ ). To note, the Gsk $3 \alpha^{f /+} \beta^{f /+} ; \alpha$-Cre retina did not display an excess of dRGCs (data not shown). Interestingly, the increase in numbers of dRGCs is not associated with a significant reduction in the number of RGCs in the ganglion cell layer (GCL), referred to as orthotopic RGCs (oRGCs; Fig. 3C).

Because of their low number in control retina ( $2 \%$ of RGCs), dRGCs have been poorly characterized with very few markers identified, such as Brn3a (Nadal-Nicolás et al., 2012, 2014). Immunostaining on sections and flatmounted retinas with additional RGC marker antibodies revealed that dRGCs in Gsk3 $\alpha^{f /+} \beta^{f / f} ; \alpha$-Cre retina were also positive for Rbpms (Rodriguez et al., 2014), confirming their increased number in the INL compared with controls (Fig. 3D,E). Similar results were observed with Islet1 labeling (Extended Data Fig. 3-1A; BejaranoEscobar et al., 2015). Previous work showed that the number of dRGCs varies by retinal domain (Dräger and Olsen, 1980). Counting of Rbpms- or Brn3a-positive dRGCs on Gsk3 $\alpha^{f /+} \beta^{f / f} ; \alpha$-Cre flat-mounted retinas did not show any significant differences in their distribution among the dorsal, ventral, nasal, and temporal regions (Extended Data Fig. 3-1B). Finally, Brn3a-positive dRGCs did not express markers of other INL neurons such as choline-acetyltransferase (CHAT; amacrine cells) or calbindin (horizontal and amacrine cells; Extended Data Fig. 3-2). Altogether, our results strongly support a ganglion cell identity of the displaced Brn3a- and RPBMS-positive cells located in the INL of Gsk3 $\alpha^{f /+} \beta^{f / f} ; \alpha$-Cre.

To test whether dRGCs in Gsk3 mutant mice were produced during the same developmental time window as oRGCs, we performed pulse chase experiments by injecting EdU at E12.5, at the peak of RGC birth. Retinal sections 
A

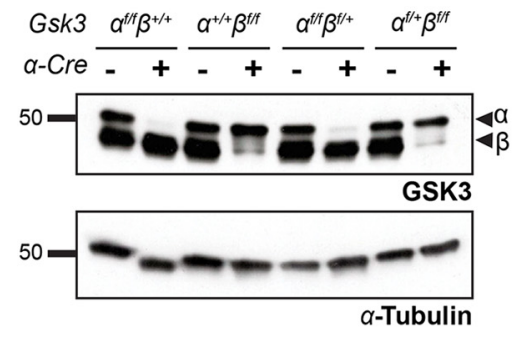

C
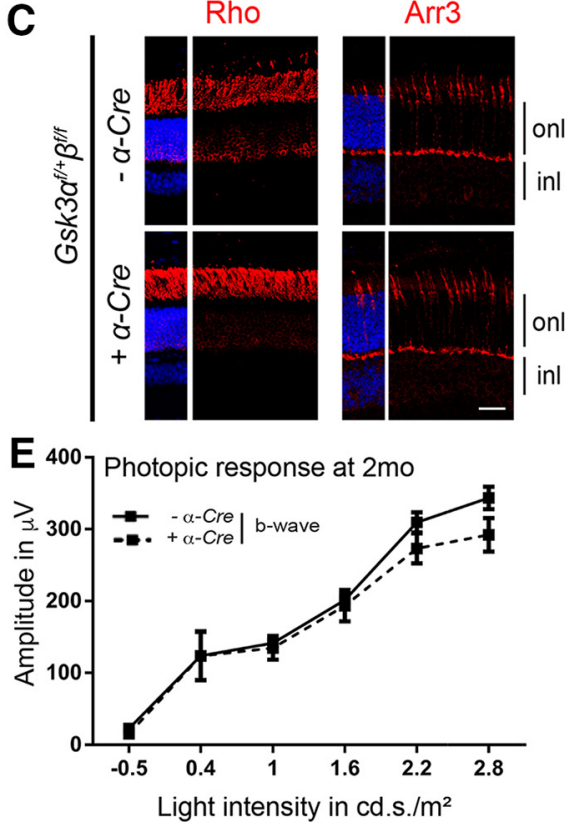

B

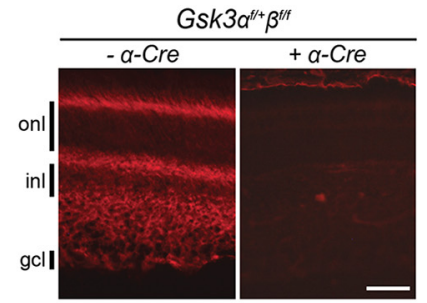

D
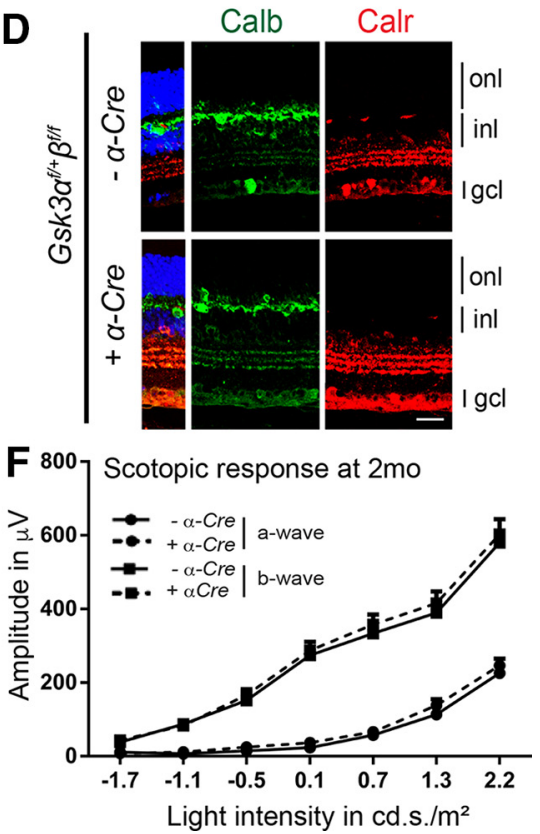

Figure 2. One allele of either Gsk3 $\alpha$ or Gsk3 $\beta$ is sufficient for the development of a functional retina. $\boldsymbol{A}$, Immunoblot analysis of protein extracts from 2-month-old animals with a different combination of Gsk3 $\alpha$ and Gsk3 $\beta$ floxed alleles (Gsk3 $\alpha^{f / f} \beta^{+/+}$, Gsk3 $\alpha^{+/+}$ $\beta^{f / f}$, Gsk $3 \alpha^{f /+} \beta^{f / f}$, or Gsk3 $\alpha^{f / f} \beta^{f /+}$ ) with or without Cre recombinase using anti-pan GSK3 antibody (recognizing both isoforms) reveals decreased expression of GSK3 $\alpha$ or GSK3 $\beta$ (arrowheads). $\alpha$-Tubulin is used as a loading control. $\boldsymbol{B}$, IHC on 2-month-old retinal sections from control and Gsk3 $\alpha^{f /+} \beta^{f / f} ; \alpha$-Cre retinas with or without Cre recombinase using anti-GSK3 $\beta$ antibody (red) showing ubiquitous Gsk3 $\beta$ expression in all retinal layers, whereas its expression is lost in the Cre-expressing retina. $\mathbf{C}$, Expression of only one Gsk3 allele (Gsk3 $\alpha$ ) is sufficient for proper photoreceptor development. IHC using anti-rhodopsin (Rho; red) and anti-Cone arrestin (Arr3; red) antibodies to label rod and cone photoreceptors, respectively. D. Expression of only one Gsk3 allele (Gsk3 $\alpha$ ) is sufficient for proper interneuron development. IHC using anti-Calretinin (Calr; green) and anti-calbindin (Calb; red) antibodies to label horizontal and amacrine cells, respectively. onl, outer nuclear layer; inl, inner nuclear layer; gcl, ganglion cell layer. Scale bar, $20 \mu \mathrm{m}$. $\boldsymbol{E}, \boldsymbol{F}$, ERG recording in 2-month-old Gsk3 $\alpha^{f /+} \beta^{f / f} ; \alpha$-Cre animals and littermate controls. Photopic (cones; $\boldsymbol{E}$ ) and scotopic (rods; $\boldsymbol{F}$ ) responses in Gsk3 $\alpha^{f /+} \beta^{f / f} ; \alpha$-Cre animals are similar to those in controls. The mean \pm SEM intensity response curves of a- and bwave responses were averaged from eight biological replicates of each genotype.

from 1-month-old animals were then immunolabeled using anti-Brn3a antibody (Fig. 4A). In control and Gsk3 $\alpha^{f /+} \beta^{f / F}$; $\alpha$-Cre retina, we identified $40-50 \%$ of RGCs that were $\mathrm{Brn3a} / \mathrm{EdU}$-positive in all layers examined (GCL and INL), indicating that both $\mathrm{dRGCs}$ and oRGCs were born around the same time (Fig. 4B). We next examined whether dRGCs are overproduced during normal retinal development and eliminated later on. In this context, increased numbers of dRGCs in Gsk $3 \alpha^{f /+} \beta^{f / f} ; \alpha$-Cre retinas could result from a defect in dRGC riddance occurring during the first 2 postnatal weeks, a period of developmental cell death in the retina (Young, 1984; Galli-Resta, 1996). At P0, the number of Brn3a-positive oRGCs was similar between littermate control and Gsk3 $\alpha^{f /+} \beta^{f / f} ; \alpha$-Cre retinas (Fig. $4 C, D)$. In contrast, the proportion of Brn3a-positive cells located in the inner part of neuroblastic layer, corresponding presumably to dRGCs, was much lower in control retinas $(6 \pm 0.1 \%)$ compared with $G s k 3 \alpha^{f /+}$ $\beta^{f / f} ; \alpha$-Cre retinas $(30 \pm 1.4 \%)$. Thus, dRGCs are not overproduced and eliminated postnatally in control retina. Our results demonstrate that dRGCs are generated during early waves of retinogenesis in Gsk3 $\alpha^{f /+} \beta^{f / f} ; \alpha$-Cre retina and strongly suggest that GSK3s play a role in restricting their numbers during normal retinal development.

\section{dRGCs produced in the absence of either Gsk3 $\alpha$ or} Gsk3 $\beta$ project to accessory visual system circuitry

Previous studies in birds and reptiles have reported that dRGCs could be responsible for optokinetic nystagmus, as they mostly project to the accessory optic nuclei (AOSs; Cook and Podugolnikova, 2001), which are critical for non-image-forming circuit and image stabilization 
A

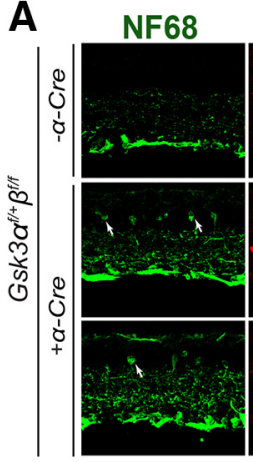

C

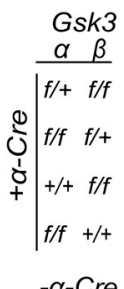
Brn3

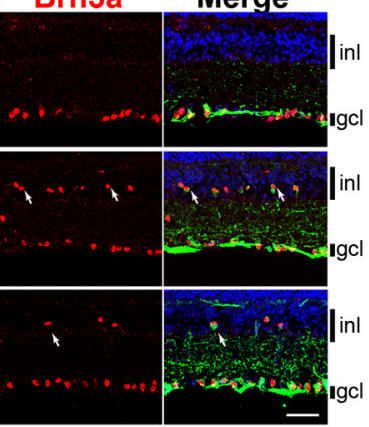

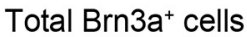
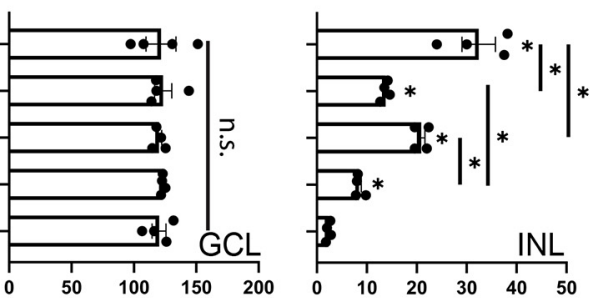

Cells/section
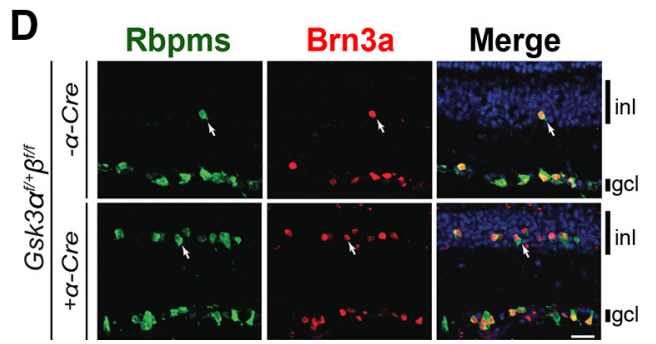

B
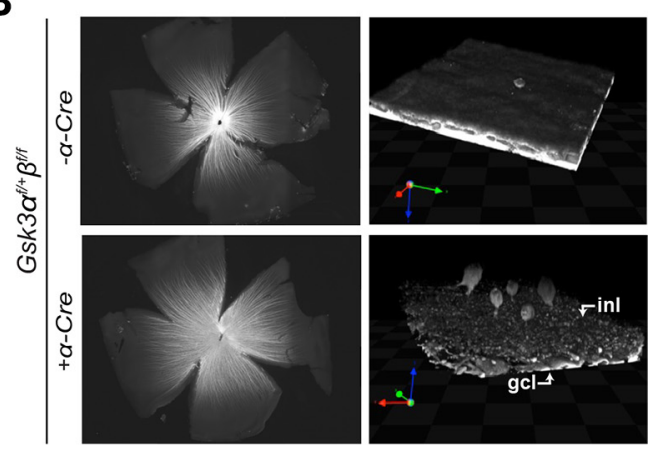

$\mathrm{Brn}^{3} \mathrm{a}^{+} \mathrm{dRGC}$

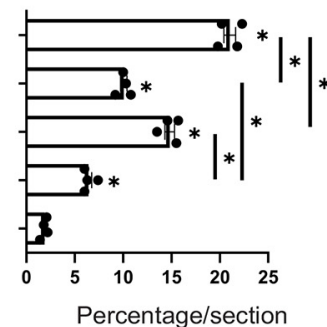

E

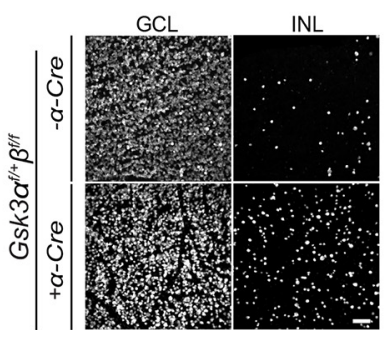

Figure 3. Gradual loss of Gsk3 $\alpha$ and/or Gsk3 $\beta$ leads to an increased number of Brn3a-positive retinal ganglion cells displaced in the INL of adult retina. A, Brn3a (red) and NF68 (green) IHC on 2-month-old Gsk3 $\alpha^{f /+} \beta^{f / f} ; \alpha$-Cre mouse retinas reveals the presence of supernumerary dRGCs (arrows) in the INL of Gsk3 $\alpha^{f /+} \beta^{f / f} ; \alpha$-Cre compared with littermate controls. Top panel represents control retinas, middle panel represents a peripheral retinal area, and bottom panel represents a more central area. Scale bar, $20 \mu \mathrm{m}$. $\boldsymbol{B}$, dRGCs send their axons into the optic nerve. Visualization of dRGCs after 3D reconstruction of 2-month-old flat mounted retinas of control and Gsk3 $\alpha^{f /+} \beta^{f / f} ; \alpha$-Cre animals following retrograde labeling with rhodamine-dextran applied onto the optic nerve. inl: inner nuclear layer, gcl: ganglion cell layer. C, Gradual loss of Gsk3 $\alpha$ and Gsk3 $\beta$ alleles (Gsk3 $\alpha^{f / f} \beta^{+/+}$, Gsk $3 \alpha^{+/+} \beta^{f / f}$, Gsk3 $\alpha^{f /+} \beta^{f / f}$ or Gsk3 $\alpha^{f / f} \beta^{f /+}$ ) leads to a gradual increase of Brn3a-positive RGCs located to the INL, with the highest number observed in Gsk3 $\alpha^{f /+} \beta^{f / f} ; \alpha$-Cre animals. Left histograms represent counting of the total number of Brn3a-positive cells per section located in the GCL (left) or in the INL (middle). Right histogram represents the percentage of the dRGCs among the total number of Brn3a-positive cells per section for each combination. Mean \pm SEM values are presented from four biological replicates. A nonparametric Mann-Whitney $U$ test was applied. ${ }^{*} p \leq 0.05 ; n s$, non significant. D, Brn3a (red) and Rbpms (green) IHC on 2-month-old mouse retinas reveal the coexpression of these two RGC markers (dRGCs, arrows) in the INL of both Gsk3 $\alpha^{f /+} \beta^{f / f} ; \alpha$-Cre dRGCs and in littermate controls. Scale bar, $20 \mu \mathrm{m}$. E, Flat-mounted retinas from Gsk3 $\alpha^{f /+} \beta^{f / f} ; \alpha$-Cre and littermate controls labeled with anti-Rbpms antibody demonstrated the large number of Rbpms-positive dRGCs in the INL of Gsk3 $\alpha^{f /+} \beta^{f / f} ; \alpha$-Cre mice. Scale bar, $50 \mu \mathrm{m}$. See Extended Data Figure 3-1 for Islet1 and Rbpms colocalization used to complete dRGC characterization and the repartition of the dRGCs in the retina. See Extended Data Figure 3-2, showing that Brn3a-positive dRGCs are not positive for amacrine or horizontal cell markers.

(Simpson, 1984). To test whether dRGCs in Gsk3 mutants project into specific visual nuclei in the brain, including the AOS, we traced the total pool of RGCs, including dRGCs, with CTB. Bilateral injection of CTB, coupled to either an Alexa Fluor-555 or Alexa Fluor-647 followed by 3D imaging, allowed us to trace both ipsilateral and contralateral projecting axons. We first confirmed that CTB injections indeed marked the dRGCs based on flat-mounts of retinas after Brn3a and NF68 immunolabeling (Extended Data Fig. 5-1). To visualize the entire visual projection network, we conducted whole-brain clearing using iDISCO + followed by light-sheet fluorescent imaging and 3D reconstruction (Fig. $5 A$ ). The complete loss of Gsk3 $\beta$ yielded a large increase in ipsilateral projecting RGCs in one of the three nuclei composing the AOS, the MTN (Simpson, 1984). This terminal nucleus is the main component of the AOS, reacting best to either upward or downward movement and mediating the optokinetic nystagmus, which is critical for image stabilization (Yonehara et al., 2009). Calculation of the signal intensity ratio between the ipsilateral and contralateral MTN demonstrated a significant increase in RGC projections into the ipsilateral MTN in retinas with Gsk3 $\beta$ deletion (Fig. $5 B$ ). 
A
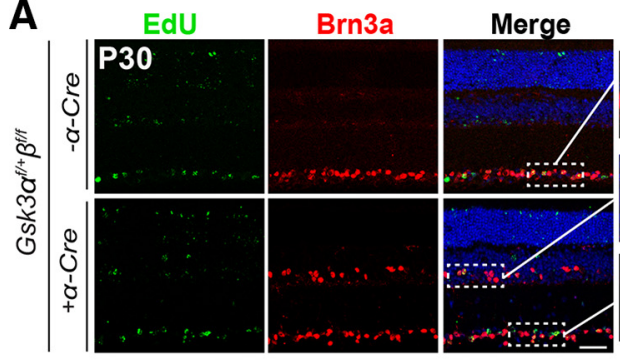

C

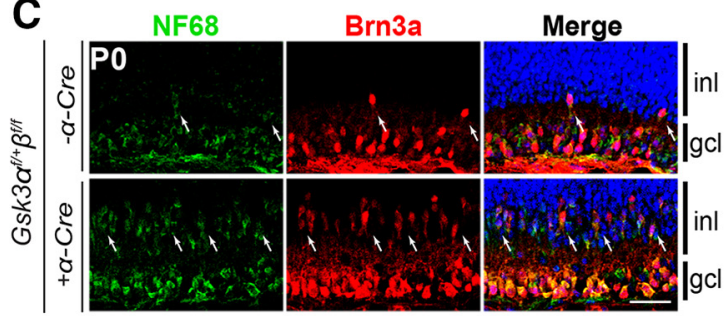

B
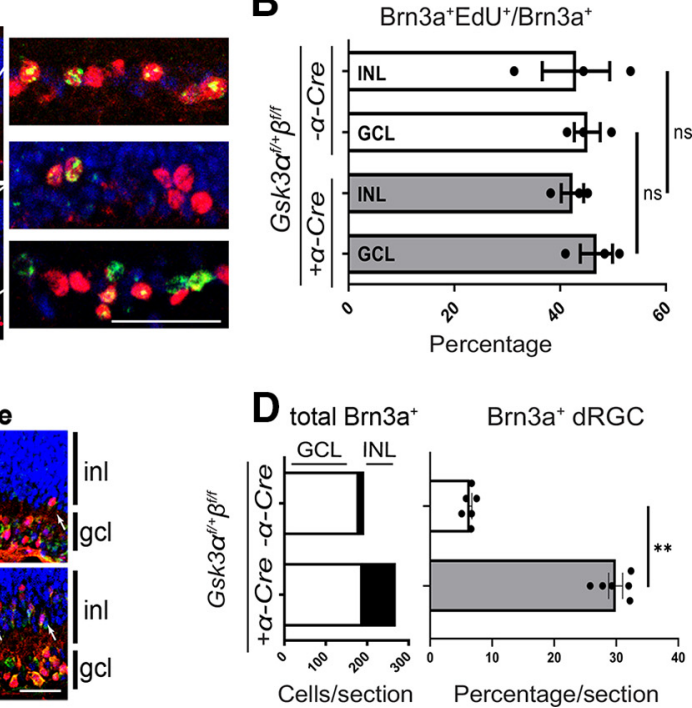

Figure 4. dRGCs are produced in the same differentiation wave as oRGC located in the GCL. $\boldsymbol{A}$, EdU-positive cells (green) and Brn3a-positive cells (red) were found both in the GCL and the INL of 30-day-old Gsk3 $\alpha^{f /+} \beta^{f / f} ; \alpha$-Cre animals after a single injection of EdU at E12.5. B, Percentage of EdU- and Brn3a-positive cells located either in the GCL or in the INL among total number of Brn3a-positive cells. Mean \pm SEM values are presented from three to four biological replicates. A nonparametric Mann-Whitney $U$ test was applied. ns, non significant. C, Brn3a (red) and NF68 (green) immunostaining on P0 mouse retinas revealed that a large number of dRGCs were already present in Gsk3 $\alpha^{f /+} \beta^{f / f} ; \alpha$-Cre mice, but were fewer in littermate controls (white arrows). $\boldsymbol{D}$, Left stacked histogram represents the counting of the total number of Brn3a-positive cells per section located in the GCL (white bars) and the INL (black bars) of Gsk3 $\alpha^{f /+} \beta^{f / f} ; \alpha$-Cre retinas. Right histogram represents the percentage of the dRGCs among the total number of Brn3a-positive cells per section. Mean \pm SEM values are presented from six biological replicates. A nonparametric Mann-Whitney $U$ test was applied. ${ }^{* *} p \leq 0.01$. inl, inner nuclear layer; gcl, ganglion cell layer. Scale bar, $20 \mu \mathrm{m}$.

This result suggests that excess dRGCs might participate in the non-image-forming circuit.

\section{Whole-transcriptome analysis suggests that dRGCs in GSK3 mutant retinas are direction-selective ganglion cells}

We next performed transcriptome analysis using RNASeq to identify molecular changes in adult Gsk3 $\alpha^{f /+} \beta^{f / f}$; $\alpha$-Cre retina and to better characterize dRGCs. Retinas from Gsk3 $\alpha^{f /+} \beta^{f / f}$ mice were used as controls. Gene-level analysis revealed 111 DEGs using filtering criteria of $\mathrm{FC}=1.5$, with an FDR cutoff of $\leq 0.05$ and a minimum mean expression value of $1 \mathrm{FPKM}$ in at least one of the two experimental groups (Fig. 6A, Extended Data Fig. 61). Pathway analysis of DEGs revealed several statistically significant overrepresented pathways (Extended Data Fig. $6-2)$. Biological processes and molecular function pathways included 48 DEGs; of these, 33 genes were expressed in RGCs based on published whole-transcriptomic data from purified RGCs (for a total 69 RGC-expressed genes among the 111 DEGs; see Fig. 8B, stars; Sajgo et al., 2017). The dominance of RGC-expressed genes in our dataset is consistent with the high number of dRGCs observed in the Gsk3 $\alpha^{f /+} \beta^{f / f} ; \alpha$-Cre retina.

Among interesting candidates dysregulated in the biological processes and molecular function pathways (Fig. 6B, Extended Data Fig. 6-2), we identified Chrna2, Chrna5, Chrna7, and Chrnb4 encoding for postsynaptic subunits of the nicotinic cholinergic receptor. With the exception of Chrna2, all other genes are upregulated in
Gsk3 $\alpha^{f /+} \beta^{f / f} ; \alpha$-Cre retina. Most retinal ganglion cells express nicotinic receptors (Kay et al., 2011; Rousso et al., 2016). Among other potentially relevant genes, the Grik3 gene product belongs to the kainate family of glutamate receptors functioning as ligand-activated ion channels. In direction-selective ganglion cells (DSGCs), glutamate is proposed to be the main source of excitation (Sweeney et al., 2014, 2019). Finally, Cartpt, encoding for the preprotein CART (Cocaine- And Amphetamine-Regulated Transcript Protein) that was upregulated in Gsk3 $\alpha^{f /+} \beta^{f / f} ; \alpha$-Cre retina, was validated by qRT-PCR (Fig. $6 C$ ). Cartpt is specifically expressed in direction-selective RGCs (DS-RGCs; Rousso et al., 2016), suggesting that dRGCs (or at least a subset) in the Gsk3 $\alpha^{f /+} \beta^{\text {fff; }} ; \alpha$-Cre retina might be DS-RGCs. In support of this hypothesis, we found some dRGCs in Gsk3 $\alpha^{f /+}$ $\beta^{f f f} ; \alpha$-Cre and littermate control retinas that were positive for the transcription factor Tbr2, which has been described as being essential for RGC specification participating in nonimage-forming visual circuits (Fig. 6D; Simpson, 1984; Yonehara et al., 2009). A small subset of dRGCs also expressed Foxp2, a transcription factor involved in DS-RGC differentiation in mice (Fig. 6D; Sato et al., 2017). These two factors were expressed in a mutually exclusive way in Rpbmspositive dRGCs, suggesting that dRGCs in Gsk $3 \alpha^{f /+} \beta^{f / f} ; \alpha$-Cre might encompass several subtypes.

\section{Optomotor response is impaired in GSK3 mutant}

Given that DS-RGCs are reported to drive the OMR by projecting mainly into the contralateral AOS (Simpson, 

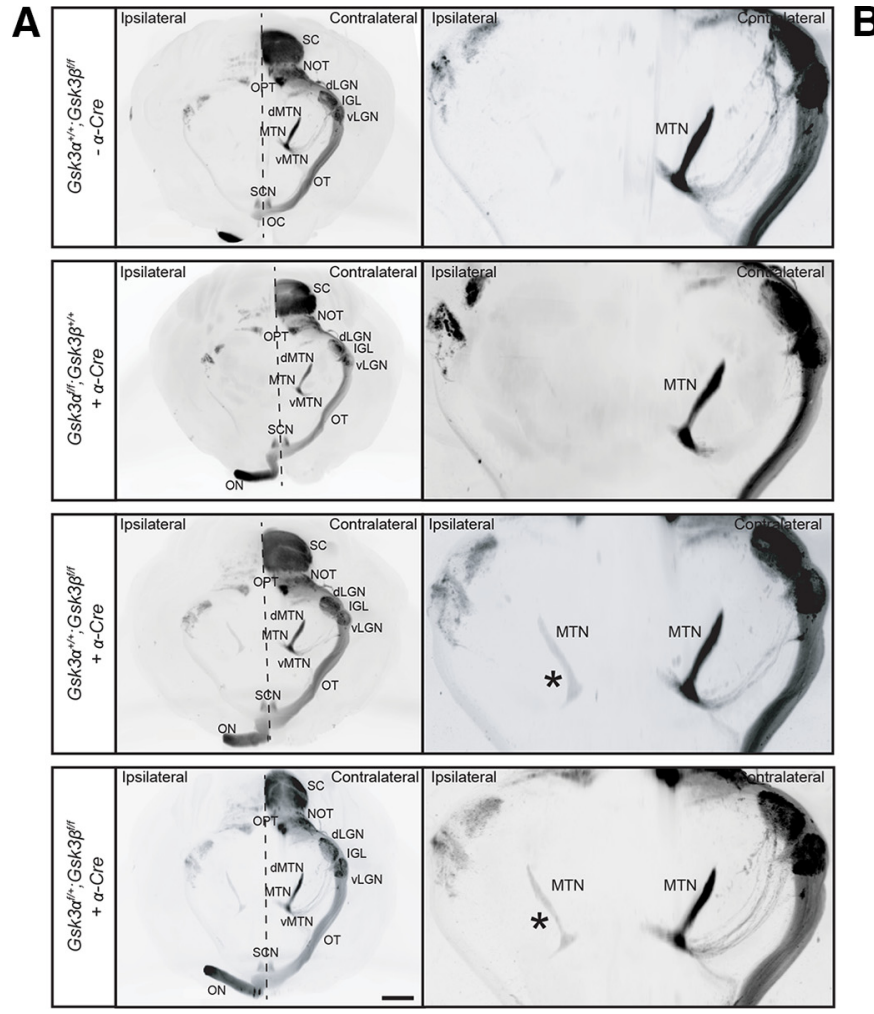

B

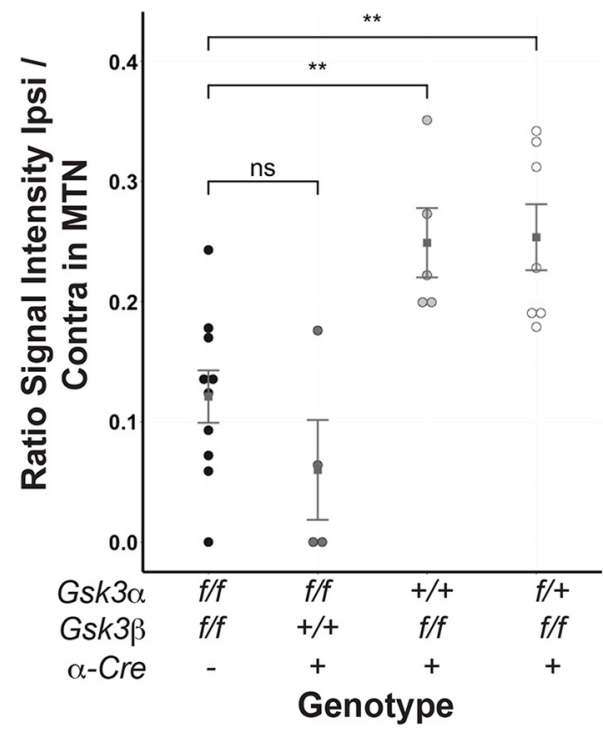

Figure 5. Lack of Gsk3 $\beta$ results in RGC projections into the ipsilateral medial terminal nucleus. $\boldsymbol{A}$, All panels are light sheet fluorescence microscopy of solvent-cleared adult brain from control, Gsk3 $\alpha^{f / f} \beta^{+/+} ; \alpha$-Cre, Gsk $3 \alpha^{+/+} \beta^{f / f} ; \alpha$-Cre, and Gsk3 $\alpha^{f /+} \beta^{f / f} ; \alpha-C r e$ animals after intravitreal injection of CTB coupled to either Alexa Fluor-555 or Alexa Fluor-647. Ipsilateral projections of RGCs into the MTN were observed in the absence of Gsk3 $\beta$ expression. NOT, Nucleus of optic tract; LGN, lateral geniculate nucleus; vLGN, ventral LGN; IGL, intergeniculate leaflet; OPT, olivary pretectal nucleus; MTN, medial terminal nucleus; dMTN, dorsal MTN; vMTN, ventral MTN; OT, optic tract; SCN, suprachiasmatic nucleus; ON, optic nerve; SC, superior colliculus. Scale bar, 1 mm. *Ipsilateral MTN. B, Quantification of the signal intensity ratio between ipsilateral and contralateral MTN in controls and Gsk3 mutants (including Gsk3 $\alpha^{f / f} \beta^{+/+} ; \alpha$-Cre, Gsk3 $\alpha^{+/+} \beta^{f / f} ; \alpha$-Cre, and Gsk3 $\alpha^{f /+} \beta^{f / f} ; \alpha$-Cre). A nonparametric Mann-Whitney $U$ test was applied. ns, non significant. ${ }^{* *} p \leq 0.01$. See Extended Data Figure 5-1 for costaining of the CTB-positive cells with Brn3a and NF68.

1984; Yonehara et al., 2009), we tested the OMR of Gsk3 $\alpha^{f /+} \beta^{f / f} ; \alpha$-Cre mice. The OMR indices (T_correct/ $T$ incorrect) were calculated from three trials at contrasts $100 \%$ and $50 \%$ (Fig. 6E). At 100\% contrast, the OMR indices were significantly reduced in Gsk $3 \alpha^{f /+} \beta^{f / f} ; \alpha$-Cre mice compared with controls at $0.05,0.15$, and $0.25 \mathrm{cpd}$. The maximum OMR index was observed at $0.15 \mathrm{cpd}$ in controls, whereas it reached a maximum at 0.1 in Gsk3 $\alpha^{f /+}$ $\beta^{f / f} ; \alpha$-Cre mice. At $50 \%$ contrast, the OMR indices were also significantly reduced in Gsk3 $\alpha^{f /+} \beta^{f / f} ; \alpha$-Cre mice compared with controls, but to a larger extent between 0.05 and $0.3 \mathrm{cpd}$. The maximum OMR index was observed at $0.15 \mathrm{cpd}$ in both controls and $\mathrm{Gsk} 3 \alpha^{f /+} \beta^{f / f}$; $\alpha$-Cre mice. Altogether, these results demonstrate an impaired OMR in Gsk3 $\alpha^{f /+} \beta^{f / f} ; \alpha$-Cre mice. These data, together with our transcriptomic and axonal projection analyses, suggest that at least a subset of dRGCs expressing only one allele of Gsk3 $\alpha$ are DS-RGCs.

\section{Discussion}

In this study, we report that complete loss of GSK3 in retinal progenitors leads to microphthalmia in adult mice with severe morphological defects. Such a severe phenotype was not observed anymore when only one Gsk3 $\alpha$ or Gsk3 $\beta$ allele was expressed, confirming the functional redundancy of these two genes. Our results implicate GSK3s as the first reported determinants of dRGCs during retinal histogenesis. Indeed, we show that mouse retinas with only one allele of Gsk3 exhibit an excessive number of dRGCs. The concomitant large increase of axonal projections to the ipsilateral MTN, our RNA-Seq data, and optomotor response tests, have led us to propose that these dRGCs are involved in the detection of image motion direction.

In pigmented wild-type mouse retina, dRGCs in the INL are a very rare and poorly described type of cell, which represents only 2\% of RGCs (Balkema and Dräger, 1990; Doi et al., 1994; Dräger and Olsen, 1980; Nadal-Nicolás et al., 2014). It is therefore striking that dRGC numbers increase up to $20 \%$ of RGCs when a single copy of Gsk3 $\alpha$ is present in retinal progenitors. To our knowledge, such a high number of dRGCs has never been reported in a transgenic/mutant animal. A previous study hypothesized that dRGCs are misplaced in the INL because of an ontogenic aberration rather than representing an independent class of RGCs (Buhl and Dann, 1988; Doi et al., 1994). Indeed, differential cell adhesion plays a key role in the 
A

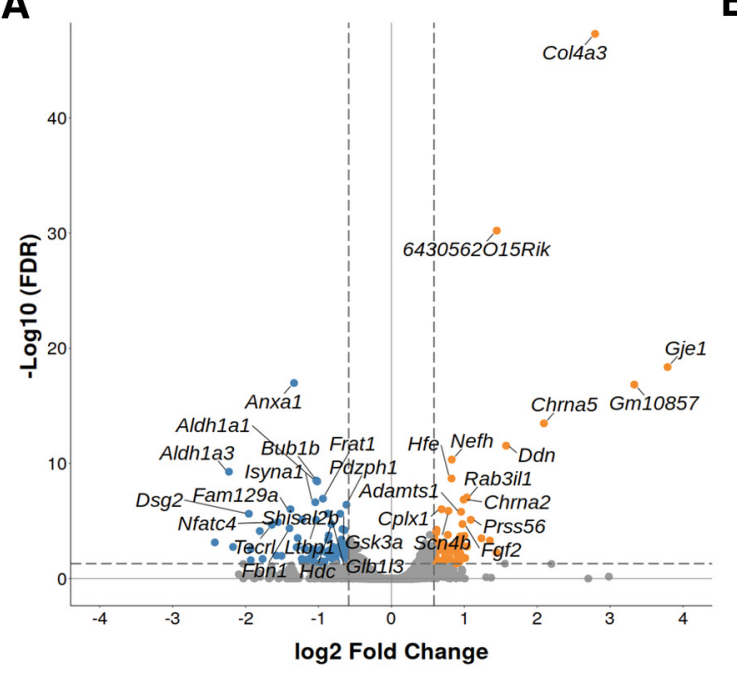

B

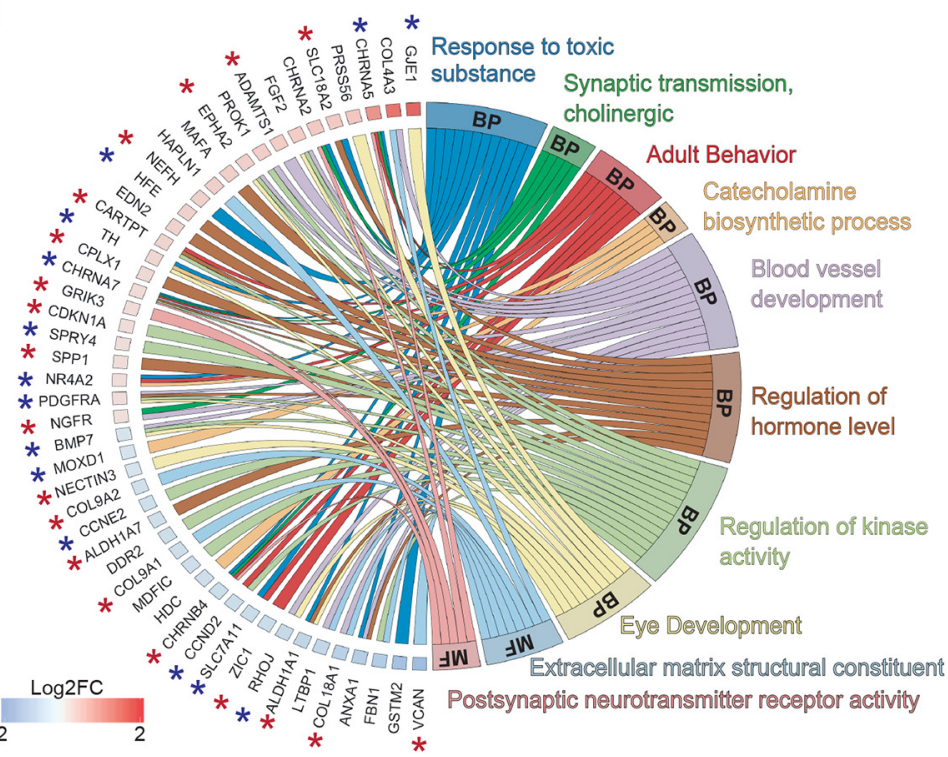

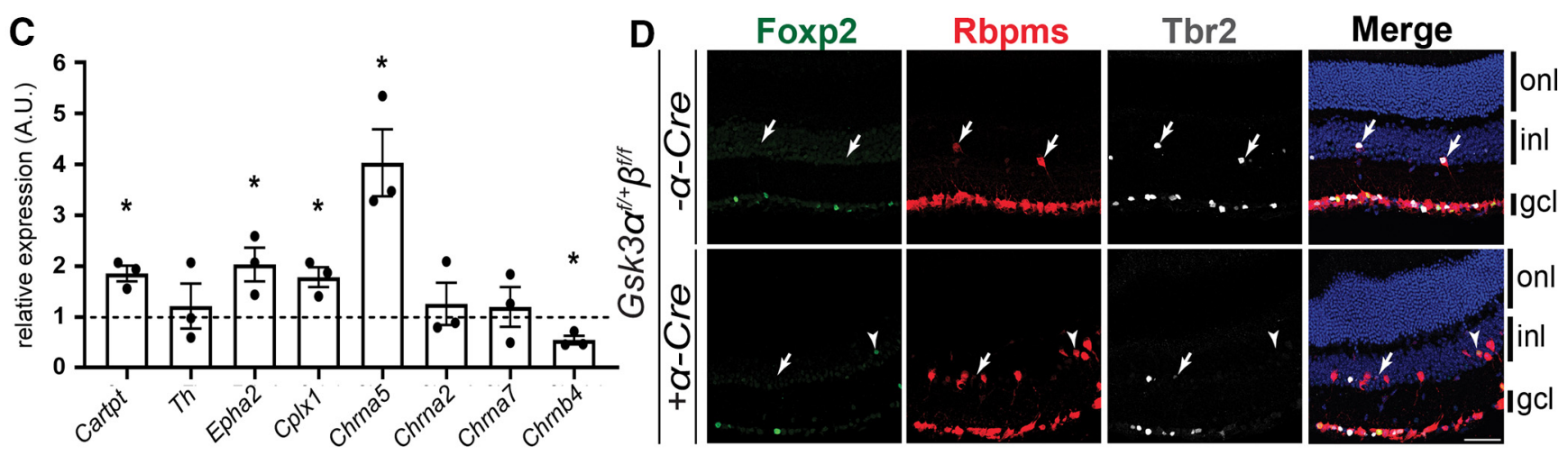

E

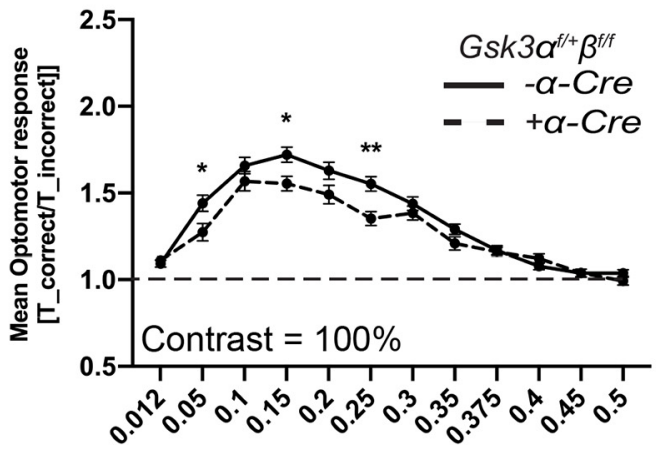

Spatial frequency [cyc $/^{\circ}$ ]

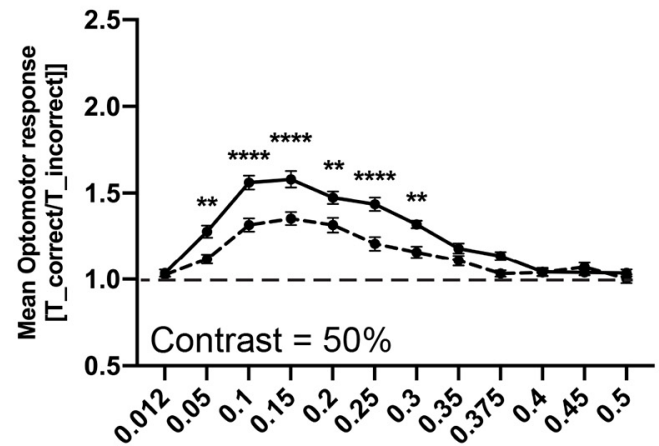

Spatial frequency [cyc/ $/^{\circ}$ ]

Figure 6. Whole-transcriptome meta-analysis suggests that dRGCs in the Gsk3 $\alpha^{f /+} \beta^{f / f} ; \alpha$-Cre retina are DS-RGCs. $\boldsymbol{A}$, Volcano plot representation of differentially expressed genes between Gsk3 $\alpha^{f /+} \beta^{f / f} ; \alpha$-Cre and control retinas plotted on the $x$-axis (log2 scale). FDR-adjusted significance is plotted on the $y$-axis. Orange and blue dots indicate significantly upregulated and downregulated genes in Gsk3 $\alpha^{f /+} \beta^{f / f} ; \alpha$-Cre retinas, respectively. Vertical dashed lines represent $\mathrm{FC}=1.5$. Horizontal dashed line represents $\mathrm{FDR}=0.05$. B , Chord plot representation of DEGs related to GO (Gene Ontology) annotations belonging to either molecular function (MF) or biological process (BP). Overlaps in GO annotation among genes within each category are visualized. *Genes expressed in previously published purified RGCs (blue, slightly expressed genes in RGCs between 1 and 5 FPKM; red, highly expressed genes in RGCs > 5 FPKM). C, qRT-PCR validation of selected DEGs identified by RNA-Seq analysis. Differential expression analysis by qRTPCR of Cartpt, Th, Epha2, Cplx1, Chrna5, Chrna2, Chrna7, and Chrnb4 in Gsk3 $\alpha^{f /+} \beta^{f / f}$; $\alpha$-Cre retinas at 2 months of age, relative to levels in littermate control retinas. All values are expressed as the mean \pm SEM from three biological replicates. A nonparametric Mann-Whitney $U$ test was applied, ${ }^{*} p \leq 0.05$. $D$, IHC on 2-month-old mouse retinas reveals the presence of a subset of dRGCs (Rbpms-positive dRGCs, red) in Gsk3 $\alpha^{\overline{f /}+} \beta^{f / f} ; \alpha$-Cre expressing either the transcription factor Tbr2 (gray) or Foxp2 (green). Arrows 
continued

indicate Tbr2 and Rbpms-positive dRGCs; arrowheads represent Foxp2 and Rbpms-positive dRGCs. onl, outer nuclear layer; inl, inner nuclear layer; gcl, ganglion cell layer. Scale bar, $50 \mu \mathrm{m}$. $\boldsymbol{E}$, The mean OMR indices ( \pm SEM) are plotted as a function of spatial frequency for each genotype ( $n=13$ for Gsk3 $\alpha^{f /+} \beta^{f / f}$ and 18 for Gsk3 $\alpha^{f /+} \beta^{f / f} ; \alpha$-Cre genotype). The baseline (1; dashed line) represents unspecific head movements and no response to the stimulus. OMR at $100 \%$ and $50 \%$ contrast in Gsk3 $\alpha^{f /+} \beta^{f / f}$; $\alpha$-Cre mice (dashed line) and controls (black line). A Grubbs' test was performed at $5 \%$ to remove outliers followed by two-way ANOVA: ${ }^{\star} p \leq 0.05,{ }^{* *} p \leq 0.01,{ }^{* \star} p \leq 0.001$. Extended Data Figure 6-1 for the hierarchical clustering of the DEGs. See Extended Data Figure 6-2 for pathway analysis results.

sorting and migration of retinal cells in their appropriate layers, especially for RGCs. One can therefore hypothesize that enhanced dRGCs in mice with a single copy of Gsk3 is the consequence of increased aberration events. This hypothesis could be supported by our RNA-Seq data showing the upregulation of genes coding for collagen subunits (Col18a1, Col4a3, Col9a1, and Col9a2) and extracellular matrix proteins in the Gsk $3 \alpha^{f /+} \beta^{f / f} ; \alpha$-Cre retina, which could favor migration defects. Noticeably, if it were the case, the increase in dRGCs should be accompanied by a decrease in oRGCs. However, we found that the number of oRGCs in the GCL is unaltered, strongly suggesting that RGCs in the INL of mice with a single copy of Gsk3 represent a specific subtype of RGCs. In support of this, topographic and quantitative analyses of RGCs in albinos and pigmented rats indicate that dRGCs are not misplaced by ontogenic mistakes but indeed represent a specific subpopulation of RGCs (Nadal-Nicolás et al., 2014). GSK3 $\beta$ was previously shown to be involved in neural cell fate decision by controlling the timing of the activity of bHLH transcription factors, such as NeuroD or Neurog2 (Moore et al., 2002; Li et al., 2012). If dRGCs are not produced following ontogenic aberrations but are instead determined by a proper genetic program, it would be interesting to identify the transcription factors involved and seek for any regulation by GSK3s. Along this line, further studies would allow a better understanding of whether the excess of dRGCs occurs only because of an expanded pool of normally occurring dRGCs or whether their presence is also a consequence of an aberrant migration during retinal development when GSK3s are not fully active. New sequencing technology such as singlecell RNA-Seq would definitively be an asset to shed more light on specific markers for dRGCs and to identify key players of dRGC specification/differentiation. As a distinct cell type, scRNA-Seq analysis following high-depth sequencing should highlight a cell cluster in t-distributed stochastic neighbor embedding plots in retina with only one Gsk3 $\alpha$ allele expressed corresponding to dRGCs. The interest and power of such approach has already been demonstrated for RGC characterization (Rheaume et al., 2018). Whole-transcriptome analysis at early time points when RGCs are produced might also complete such analysis.

In reptiles, amphibians, and birds, only dRGCs project into the MTN, whereas in mammals only oRGCs have been reported as projecting into the MTN (Fite et al., 1981; Dann and Buhl, 1987; Krause et al., 2014). Our results obtained from anterograde labeling clearly demonstrated a large increase in ipsilateral MTN projections in the absence of Gsk3 $\beta$, whereas it was absent or very dim in control animals. Although this strongly suggests that excess dRGCs in mutant mice are causing this phenotype, we cannot exclude the possibility that mutant oRGCs also participate in these ipsilateral MTN projections. However, contralateral projections did not seem to be affected. Noticeably, however, it would be challenging to observe an increase in dRGC projections into the other areas already strongly labeled using our anterograde labeling method, especially into the dorsal lateral geniculate nucleus (dLGN) or superior colliculus (SC). We can speculate that the low number of ipsilateral MTN projections in the control condition reflects the low number of dRGCs present in the WT retina and could therefore explain why such a result had not been described so far. Altogether, our results strongly suggest that dRGCs may primarily project into the ipsilateral MTN. In mice, it has been shown by retrograde labeling from the $\mathrm{SC}$, which receives a large number of RGC projections, that dRGCs/oRGCs project to one or both SCs (Karten et al., 1977). Although challenging, similar experiments (i.e. fluorescent dye injection into the ipsilateral MTN) may allow us to discriminate whether the increased signal in the absence of Gsk3 $\beta$ originates only from dRGCs and whether these cells also project into this area in WT retina. In regard to our results, it is still unclear whether the function of GSK3 is to limit the number of dRGCs and to actively regulate their correct projection to the contralateral MTN or whether GSK3 function is limited to tightly controlling the number of dRGCs, which project thereafter to the ipsilateral MTN in a GSK3-independent manner.

Given the very low percentage of dRGCs in the control retina, their function is poorly studied in mammals. In contrast, dRGC function, projections, and topography have been investigated in bird and reptile retina (Mouritsen et al., 2004). In birds, cryptochrome-expressing dRGCs are used as a magnetic compass for orientation (Nießner et al., 2016). In European Robin birds, Erithacus rubecula, a low number of dRGCs has been identified but specifically express Cryptochrome $1 \mathrm{~b}$ only during nocturnal migration period (Nießner et al., 2016). In rodents, retrograde labeling from the optic nerve led to the identification of 16 classes of dRGCs based on the ramification levels of their dendrites as well as the dendritic field size (Pang and $\mathrm{Wu}, 2011)$. Based on dRGC dendrite projections into the inner plexiform layer, it has been proposed that most dRGCs in the WT retina are functionally more involved in retinal OFF light pathways (Pang and Wu, 2011). Similar methods applied to the Gsk3 $\alpha^{f /+} \beta^{f / f} ; \alpha$-Cre retina should shed more light on dRGC function and establish whether all the different classes are present.

As part of the AOS, the MTN receives an afferent signal from the eye and sends an efferent signal to motor 
neurons controlling the position of the eye. As such, optokinetic reflex relies on direction-specific retinal projections to the AOS. Neurons of the dorsal terminal nucleus code for horizontal stimulus, whereas neurons of the MTN code for vertical stimulus (Giolli et al., 2006; Yonehara et al., 2009). Therefore, the direction of image motion relies on DS-RGCs in the retina. The alteration of the OMR in Gsk3 $\alpha^{f /+} \beta^{f / f} ; \alpha$-Cre mice support the hypothesis that some of the supernumerary dRGCs are indeed related to motion detection. Although the number of dRGCs was drastically increased, the OMR was not increased but, on the contrary, was reduced. Such a result might be caused by the higher number of projections to the ipsilateral side instead of the contralateral one, leading to an alteration of the neuronal circuit regulating the OMR (Simpson, 1984; Yonehara et al., 2009). We also identified in Gsk $3 \alpha^{f /+} \beta^{f / f} ; \alpha$-Cre and control retinas a small subset of dRGCs, which are positive for the transcription factors Tbr2 and Foxp2, the markers for non-image-forming RGCs and DS-RGCs, respectively (Nadal-Nicolás et al., 2014). Together with the transcriptomic data (upregulation of genes such as Cartpt expressed in DS-RGCs), these results provide strong evidence suggesting that the large number of dRGCs in the Gsk3 $\alpha^{f /+} \beta^{f / f} ; \alpha$-Cre retina might indeed be DS-RGCs projecting into the MTN. It has been recently proposed that dRGCs might also be involved in predator detection by integrating overhead visual information (NadalNicolás et al., 2014). Using suitable and complementary visual tests, our genetic model could be highly valuable to complete the functional identification of the dRGCs in visual process.

Overall, our results demonstrate a critical role of GSK3s in stringently regulating the number of a rare type of dRGCs, which has been poorly described so far. Gsk3 mutant mice, with a large number of dRGCs in their retina, offer a unique and powerful model system to further study the embryonic origin, synaptic connections, and visual function of dRGCs in mammals.

\section{References}

Balkema GW, Dräger UC (1990) Origins of uncrossed retinofugal projections in normal and hypopigmented mice. Vis Neurosci 4:595604.

Bejarano-Escobar R, Álvarez-Hernán G, Morona R, González A, Martín-Partido G, Francisco-Morcillo J (2015) Expression and function of the LIM-homeodomain transcription factor Islet-1 in the developing and mature vertebrate retina. Exp Eye Res 138:2231.

Belle M, Godefroy D, Dominici C, Heitz-Marchaland C, Zelina P, Hellal F, Bradke F, Chédotal A (2014) A simple method for 3D analysis of immunolabeled axonal tracts in a transparent nervous system. Cell Rep 9:1191-1201.

Belle M, Godefroy D, Couly G, Malone SA, Collier F, Giacobini P, Chédotal A (2017) Tridimensional visualization and analysis of early human development. Cell 169:161-173.e12.

Buhl EH, Dann JF (1988) Morphological diversity of displaced retinal ganglion cells in the rat: a lucifer yellow study. J Comp Neurol 269:210-218.

Chen Y, Lun ATL, Smyth GK (2014) Differential expression analysis of complex RNA-seq experiments using edgeR. In: Statistical analysis of next generation sequence data, (Datta S, Nettleton DS, eds). New York:Springer.
Cole AR (2012) GSK3 as a sensor determining cell fate in the brain. Front Mol Neurosci 5:4-10.

Cook JE, Podugolnikova TA (2001) Evidence for spatial regularity among retinal ganglion cells that project to the accessory optic system in a frog, a reptile, a bird, and a mammal. Vis Neurosci 18:289-297.

Dann JF, Buhl EH (1987) Retinal ganglion cells projecting to the accessory optic system in the rat. J Comp Neurol 262:141-158.

Dobin A, Davis CA, Schlesinger F, Drenkow J, Zaleski C, Jha S, Batut P, Chaisson M, Gingeras TR (2013) STAR: ultrafast universal RNA-seq aligner. Bioinformatics 29:15-21.

Doble BW, Woodgett JR (2003) GSK-3: tricks of the trade for a multitasking kinase. J Cell Sci 116:1175-1186.

Doble BW, Patel S, Wood GA, Kockeritz LK, Woodgett JR (2007) Functional redundancy of GSK-3alpha and GSK-3beta in Wnt/ beta-catenin signaling shown by using an allelic series of embryonic stem cell lines. Dev Cell 12:957-971.

Doi M, Imatani H, Sasoh M, Uji Y, Yamamura H (1994) Displaced retinal ganglion cells in the Chinese hamster. Jpn J Ophthalmol 38:139-143.

Dräger UC, Olsen JF (1980) Origins of crossed and uncrossed retinal projections in pigmented and albino mice. J Comp Neurol 191:383-412.

Eom TY, Jope RS (2009) Blocked inhibitory serine-phosphorylation of glycogen synthase kinase- $3 \alpha / \beta$ impairs in vivo neural precursor cell proliferation. Biol Psychiatry 66:494-502.

Espinosa L, Inglés-Esteve J, Aguilera C, Bigas A (2003) Phosphorylation by glycogen synthase kinase-3 $\beta$ down-regulates Notch activity, a link for Notch and Wnt pathways. J Biol Chem 278:32227-32235.

Fite KV, Brecha N, Karten HJ, Hunt SP (1981) Displaced ganglion cells and the accessory optic system of pigeon. J Comp Neurol 195:279-288.

Galli-Resta L, Ensini M (1996) An intrinsic time limit between genesis and death of individual neurons in the developing retinal ganglion cell layer. J Neurosci 16:2318-2324.

Giolli RA, Blanks RHI, Lui F (2006) The accessory optic system: basic organization with an update on connectivity, neurochemistry, and function. Prog Brain Res 151:407-440.

Golpich M, Amini E, Hemmati F, Ibrahim NM, Rahmani B, Mohamed Z, Raymond AA, Dargahi L, Ghasemi R, Ahmadiani A (2015) Glycogen synthase kinase-3 beta (GSK-3 $\beta$ ) signaling: implications for Parkinson's disease. Pharmacol Res 97:16-26.

Hamon A, García-García D, Ail D, Bitard J, Chesneau A, Dalkara D, Locker M, Roger JE, Perron M (2019) Linking YAP to Müller glia quiescence exit in the degenerative retina. Cell Rep 27:17121725.e6.

Hur E-M, Zhou F-Q (2010) GSK3 signalling in neural development. Nat Rev Neurosci 11:539-551.

Jacobs KM, Bhave SR, Ferraro DJ, Jaboin JJ, Hallahan DE, Thotala D (2012) GSK-3ß: a bifunctional role in cell death pathways. Int J Cell Biol 2012:930710.

Jin YH, Kim H, Oh M, Ki H, Kim K (2009) Regulation of Notch1/NICD and Hes 1 expressions by GSK-3 $\alpha / \beta$. Mol Cells 27:15-19.

Karten HJ, Fite KV, Brecha N (1977) Specific projection of displaced retinal ganglion cells upon the accessory optic system in the pigeon (Columbia livia). Proc Natl Acad Sci U S A 74:1753-1756.

Kay JN, de la Huerta I, Kim IJ, Zhang Y, Yamagata M, Chu MW, Meister M, Sanes JR (2011) Retinal ganglion cells with distinct directional preferences differ in molecular identity, structure, and central projections. J Neurosci 31:7753-7762.

Kim W-Y, Wang X, Wu Y, Doble BW, Patel S, Woodgett JR, Snider WD (2009) GSK-3 is a master regulator of neural progenitor homeostasis. Nat Neurosci 12:1390-1397.

Krause M, Distler C, Hoffmann KP (2014) Retinal ganglion cells projecting to the accessory optic system in optokinetic blind albinotic rats are direction-selective. Eur J Neurosci 40:2274-2282.

Kremer A, Louis JV, Jaworski T, Van Leuven F (2011) GSK3 and Alzheimer's disease: facts and fiction.... Front Mol Neurosci 4:17.

Kretschmer F, Kretschmer V, Kunze VP, Kretzberg J (2013) OMRarena: automated measurement and stimulation system to 
determine mouse visual thresholds based on optomotor responses. PLoS One 8:e78058.

Kretschmer F, Sajgo S, Kretschmer V, Badea TC (2015) A system to measure the optokinetic and optomotor response in mice. $J$ Neurosci Methods 256:91-105.

Li DW, Liu ZQ, Chen W, Yao M, Li GR (2014) Association of glycogen synthase kinase-3 $\beta$ with Parkinson's disease (Review). Mol Med Rep 9:2043-2050.

Li S, Mattar P, Zinyk D, Singh K, Chaturvedi C-P, Kovach C, Dixit R, Kurrasch DM, Ma Y-C, Chan JA, Wallace V, Dilworth FJ, Brand M, Schuurmans C (2012) GSK3 temporally regulates neurogenin 2 proneural activity in the neocortex. J Neurosci 32:7791-7805.

Liao Y, Smyth GK, Shi W (2014) FeatureCounts: an efficient general purpose program for assigning sequence reads to genomic features. Bioinformatics 30:923-930.

Livak KJ, Schmittgen TD (2001) Analysis of relative gene expression data using real-time quantitative PCR and the $2^{-\Delta \Delta C}$ method. Methods 25:402-408.

Marcus EA, Kintner C, Harris W (1998) The role of GSK3 $\beta$ in regulating neuronal differentiation in Xenopus laevis. Mol Cell Neurosci 12:269-280.

Marquardt T, Ashery-Padan R, Andrejewski N, Scardigli R, Guillemot $F$, Gruss $P$ (2001) Pax6 is required for the multipotent state of retinal progenitor cells. Cell 105:43-55.

Maurer U, Preiss F, Brauns-Schubert P, Schlicher L, Charvet C (2014) GSK-3 - at the crossroads of cell death and survival. J Cell Sci 127:1369-1378.

Medina M, Garrido JJ, Wandosell FG (2011) Modulation of GSK-3 as a therapeutic strategy on tau pathologies. Front Mol Neurosci 4:24.

Moore KB, Schneider ML, Vetter ML (2002) Posttranslational mechanisms control the timing of bHLH function and regulate retinal cell fate. Neuron 34:183-195.

Mouritsen $H$, Janssen-Bienhold $U$, Liedvogel $M$, Feenders $G$, Stalleicken J, Dirks P, Weiler R (2004) Cryptochromes and neuronal-activity markers colocalize in the retina of migratory birds during magnetic orientation. Proc Natl Acad Sci U S A 101:1429414299 .

Nadal-Nicolás FM, Jiménez-López $M$, Salinas-Navarro $M$, SobradoCalvo P, Alburquerque-Béjar JJ, Vidal-Sanz M, Agudo-Barriuso M (2012) Whole number, distribution and co-expression of Brn3 transcription factors in retinal ganglion cells of adult albino and pigmented rats. PLoS One 7:e49830.

Nadal-Nicolás FM, Salinas-Navarro M, Jiménez-López M, SobradoCalvo P, Villegas-Pérez MP, Vidal-Sanz M, Agudo-Barriuso M (2014) Displaced retinal ganglion cells in albino and pigmented rats. Front Neuroanat 8:99.

Nießner C, Gross JC, Denzau S, Peichl L, Fleissner G, Wiltschko W, Wiltschko R (2016) Seasonally changing cryptochrome 1b expression in the retinal ganglion cells of a migrating passerine bird. PLoS One 11:e0150377.

Pachenari N, Kiani S, Javan M (2017) Inhibition of glycogen synthase kinase 3 increased subventricular zone stem cells proliferation. Biomed Pharmacother 93:1074-1082.

Pang J-J, Wu SM (2011) Morphology and immunoreactivity of retrogradely double-labeled ganglion cells in the mouse retina. Invest Ophthalmol Vis Sci 52:4886-4896.

Pérezleón JA, Osorio-Paz I, Francois L, Salceda R (2013) Immunohistochemical localization of glycogen synthase and
GSK3 $\beta$ : control of glycogen content in retina. Neurochem Res 38:1063-1069.

Rheaume BA, Jereen A, Bolisetty M, Sajid MS, Yang Y, Renna K, Sun L, Robson P, Trakhtenberg EF (2018) Single cell transcriptome profiling of retinal ganglion cells identifies cellular subtypes. Nat Commun 9:3203.

Rodriguez AR, de Sevilla Müller LP, Brecha NC (2014) The RNA binding protein RBPMS is a selective marker of ganglion cells in the mammalian retina. J Comp Neurol 522:1411-1443.

Roger JE, Nellissery J, Kim D, Swaroop A (2010) Sumoylation of bZIP transcription factor NRL modulates target gene expression during photoreceptor differentiation. J Biol Chem 285:2563725644.

Roger JE, Ranganath K, Zhao L, Cojocaru R, Brooks M, Gotoh N, Veleri S, Hiriyanna A, Rachel R, Campos MM, Fariss R, Wong W, Swaroop A (2012) Preservation of cone photoreceptors after a rapid yet transient degeneration and remodeling in cone-only $\mathrm{Nrl}^{-/-}$mouse retina. J Neurosci 32:528-541.

Rousso DL, Qiao M, Kagan RD, Yamagata M, Palmiter RD, Sanes JR (2016) Two pairs of ON and OFF retinal ganglion cells are defined by intersectional patterns of transcription factor expression. Cell Rep 15:1930-1944.

Sajgo S, Ghinia MG, Brooks M, Kretschmer F, Chuang K, Hiriyanna S, Wu Z, Popescu O, Badea TC (2017) Molecular codes for cell type specification in Brn3 retinal ganglion cells. Proc Natl Acad Sci U S A 114:E3974-E3983.

Sato C, Iwai-Takekoshi L, Ichikawa Y, Kawasaki H (2017) Cell typespecific expression of FoxP2 in the ferret and mouse retina. Neurosci Res 117:1-13.

Shimizu T, Kagawa T, Inoue T, Nonaka A, Takada S, Aburatani H, Taga T (2008) Stabilized $\beta$-catenin functions through TCF/LEF proteins and the Notch/RBP- $\mathrm{J} \kappa$ complex to promote proliferation and suppress differentiation of neural precursor cells. Mol Cell Biol 28:7427-7441.

Simpson Jl (1984) The accessory optic system. Annu Rev Neurosci $7: 13-41$

Sweeney NT, Tierney H, Feldheim DA (2014) Tbr2 is required to generate a neural circuit mediating the pupillary light reflex. J Neurosci 34:5447-5453.

Sweeney NT, James KN, Nistorica A, Lorig-Roach RM, Feldheim DA (2019) Expression of transcription factors divides retinal ganglion cells into distinct classes. J Comp Neurol 527:225-235.

Walter W, Sánchez-Cabo F, Ricote M (2015) GOplot: an R package for visually combining expression data with functional analysis. Bioinformatics 31:2912-2914.

Wang B, Li Y (2006) Evidence for the direct involvement of $\beta \operatorname{TrCP}$ in Gli3 protein processing. Proc Natl Acad Sci U S A 103:33-38.

Yonehara $\mathrm{K}$, Ishikane $\mathrm{H}$, Sakuta $\mathrm{H}$, Shintani $\mathrm{T}$, Nakamura-Yonehara K, Kamiji NL, Usui S, Noda M (2009) Identification of retinal ganglion cells and their projections involved in central transmission of information about upward and downward image motion. PLoS One 4:e4320.

Young RW (1984) Cell death during differentiation of the retina in the mouse. J Comp Neurol 229:362-373.

Zhou Y, Zhou B, Pache L, Chang M, Khodabakhshi AH, Tanaseichuk O, Benner C, Chanda SK (2019) Metascape provides a biologistoriented resource for the analysis of systems-level datasets. Nat Commun 10:1523. 